\title{
Plant uptake of nitrogen adsorbed to biochars made from dairy
} manure

\author{
Leilah Krounbi ${ }^{1}$, Akio Enders ${ }^{1,2}$, John Gaunt ${ }^{2}$, Margaret Ball ${ }^{2}$ \& Johannes Lehmann ${ }^{1,3 凶}$
}

The conversion of dairy waste with high moisture contents to dry fertilizers may reduce environmental degradation while lowering crop production costs. We converted the solid portion of screw-pressed dairy manure into a sorbent for volatile ammonia $\left(\mathrm{NH}_{3}\right)$ in the liquid fraction using pyrolysis and pretreatment with carbon dioxide $\left(\mathrm{CO}_{2}\right)$. The extractable $\mathrm{N}$ in manure biochar exposed to $\mathrm{NH}_{3}$ following $\mathrm{CO}_{2}$ pre-treatment reached $3.36 \mathrm{~g} \mathrm{~N} \mathrm{~kg}^{-1}, 1260$-fold greater extractable $\mathrm{N}$ than in untreated manure biochar. Ammonia exposure was 142 -times more effective in increasing extractable $\mathrm{N}$ than immersing manure biochar in the liquid fraction containing dissolved ammonium. Radish and tomato grown in horticultural media with manure biochar treated with $\mathrm{CO}_{2}+\mathrm{NH}_{3}$ promoted up to $35 \%$ greater plant growth (dry weight) and $36-83 \%$ greater $\mathrm{N}$ uptake compared to manure biochar alone. Uptake of $\mathrm{N}$ was similar between plants grown with wood biochar exposed to $\mathrm{CO}_{2}+\mathrm{NH}_{3}$, compared to $\mathrm{N}$-equivalent treatments. The available $\mathrm{N}$ in dairy waste in New York (NY) state, if pyrolyzed and treated with $\mathrm{NH}_{3}+\mathrm{CO}_{2}$, is equivalent to $11,732-42,232 \mathrm{Mg} \mathrm{N}$ year $^{-1}$, valued at 6-21.5 million USD year ${ }^{-1}$. Separated dairy manure treated with $\mathrm{CO}_{2}+\mathrm{NH}_{3}$ can offset $23-82 \%$ of $\mathrm{N}$ fertilizer needs of $\mathrm{NY}$ State, while stabilizing both the solid and liquid fraction of manure for reduced environmental pollution.

Nitrogen $(\mathrm{N})$ management is a major challenge in agricultural systems. Nitrogen fertilizer for crops is a significant cost, while the disposal of N-rich wastes such as dairy manure, can also be costly. The shift toward sustainable farming highlights the need for opportunistic waste management able to capture nutrients from liquid waste streams and transform them into high-value, dry fertilizers that can be safely transported and traded.

Dairy manure excretion in NY State alone averages $12,821,616 \mathrm{Mg}$ per year ${ }^{1,2}$. Containing approximately $64,108 \mathrm{Mg} \mathrm{N}, 16,786 \mathrm{Mg} \mathrm{P}, 44,876 \mathrm{Mg} \mathrm{K}^{3}$, these excreted nutrients are sufficient to fertilize the state's extensive 17,321 hectares (ha) of corn production, requiring $51,360 \mathrm{Mg} \mathrm{N}$ calculated using the average application rate in $2018,96 \mathrm{~kg} \mathrm{ha}^{-14-6}$. The benefits incurred by transforming dairy waste into agronomic inputs extend to dairy farmers and grain farmers alike in NY State; a farmer growing 81 ha of corn spends 28,000 USD year ${ }^{-1}$ for $^{-}$ fertilizer $^{7}$, while a dairy farmer with 550 cows spends 25,000 USD year $^{-1}$ for manure storage ${ }^{8}$. To couple these processes, new technologies for recycling dairy waste products back into crop nutrient inputs are necessary.

While direct manure spreading has been the most common means of disposal and re-use of dairy waste, such practices can result in transport of $\mathrm{N}$ and $\mathrm{P}$ into waterways ${ }^{9,10}$, as nutrients from manure often exceed the agronomic demand ${ }^{11}$. Furthermore, manure storage for future manure application produces notable quantities of methane, a known greenhouse gas ${ }^{5,12}$. Moreover, long-distance transport of dairy manure for land application also has a number of drawbacks such as the spreading of pathogens ${ }^{13}$ and costs associated with transporting a material with a water content of $>70 \%{ }^{14}$. Alternatives to direct land application of dairy slurry are needed with less detrimental impact on the environment ${ }^{15}$, which utilize the high nutrient content of manure for agronomic purposes. Source-separation of manure followed by anaerobic digestion of the liquid portion is one alternative $e^{16-18}$ that is being adopted across farms of all scales in NY State ${ }^{19}$ and other regions.

Solid-liquid separation of manure is a first step in efficient re-use of waste nutrients ${ }^{16}$. The physical separation of manure into solid and liquid fractions (slurry) significantly lowers $\mathrm{N}$ leaching from the solid fraction ${ }^{17,18}$. Most of the inorganic $\mathrm{N}$ in dairy manure is found in the liquid portion, approximately $4400 \mathrm{mg} \mathrm{NH} \mathrm{N}_{4}-\mathrm{N} \mathrm{L}^{-117}$, and can be a significant source of $\mathrm{N}_{2} \mathrm{O}$ and $\mathrm{NH}_{3}$ emissions from lagoons ${ }^{5,20,21}$. Thus, a technology is needed for removing $\mathrm{N}$ from stored slurry and converting it into a fertilizer. We see great potential in converting the solid portion of separated dairy manure into a biological charcoal, or biochar, with high sorption properties, able to remove $\mathrm{N}$ from the liquid portion of dairy manure.

\footnotetext{
${ }^{1}$ Soil and Crop Sciences, School of Integrative Plant Science, College of Agriculture and Life Sciences, Cornell University, Ithaca, NY 14853, USA. ${ }^{2}$ bio365 LLC, Ithaca, NY 14850, USA. ${ }^{3}$ Cornell Atkinson Center for Sustainability, Cornell University, Ithaca, NY 14853, USA. ${ }^{\circledR}$ email: CL273@cornell.edu
} 


\begin{tabular}{|l|l|l|l|l|c|c|c|c|c|}
\hline Analysis & Unit & Wood biochar & Manure biochar & Slurry & $\begin{array}{l}\text { Wood biochar } \\
\mathbf{N H}_{3}+\mathbf{C O}_{2}\end{array}$ & $\begin{array}{l}\text { Manure biochar } \\
\mathbf{C O}_{2}+\mathbf{N H}_{3}\end{array}$ & $\begin{array}{l}\text { Wood } \\
\text { biochar + slurry }\end{array}$ & $\begin{array}{l}\text { Manure } \\
\text { biochar }+ \text { slurry }\end{array}$ & $\begin{array}{l}\text { Potting media } \\
(\text { peat })\end{array}$ \\
\hline Nitrogen & $\%\left(\mathrm{w} \mathrm{w}^{-1}\right)$ & $0.75 \pm 0.34 \mathrm{a}$ & $2.05 \pm 0.05 \mathrm{a}$ & $2.75 \pm 0.05 \mathrm{a}$ & $1.88 \pm 1.48 \mathrm{a}$ & $2.11 \pm 0.37 \mathrm{a}$ & $1.84 \pm 0.15 \mathrm{a}$ & $2.21 \pm 0.13 \mathrm{a}$ & $0.74 \pm 0.23 \mathrm{a}$ \\
\hline Carbon & $\%\left(\mathrm{w} \mathrm{w}^{-1}\right)$ & $90.70 \pm 0.35 \mathrm{a}$ & $44.30 \pm 0.41 \mathrm{~d}$ & $35.3 \pm 0.15 \mathrm{e}$ & $88.21 \pm 1.78 \mathrm{~b}$ & $43.68 \pm 0.75 \mathrm{~d}$ & $73.73 \pm 0.14 \mathrm{c}$ & $42.32 \pm 0.32 \mathrm{~d}$ & $25.67 \pm 1.41^{\mathrm{f}}$ \\
\hline $\mathrm{NH}_{4}{ }^{+} \mathrm{N}$ & $\left(\mathrm{mg} \mathrm{kg}^{-1}\right)$ & $4.4 \pm 4.7 \mathrm{~b}$ & $2.7 \pm 3.1 \mathrm{~b}$ & $\mathrm{ND}$ & $3083.5 \pm 143.2 \mathrm{a}$ & $3363.7 \pm 335.0 \mathrm{a}$ & $15.5 \pm 3.7 \mathrm{~b}$ & $23.7 \pm 22.2 \mathrm{~b}$ & $606.2 \pm 16.4 \mathrm{~b}$ \\
\hline $\mathrm{NO}_{3}{ }^{-} \mathrm{N}$ & $\left(\mathrm{mg} \mathrm{kg}^{-1}\right)$ & $0.8 \pm 0.1 \mathrm{~b}$ & $24.0 \pm 2.1 \mathrm{~b}$ & $\mathrm{ND}$ & $4.7 \pm 0.1 \mathrm{~b}$ & $20.6 \pm 2.7 \mathrm{~b}$ & $0.5 \pm 0.1 \mathrm{~b}$ & $2.3 \pm 1.2 \mathrm{~b}$ & $635.1 \pm 15.2 \mathrm{a}$ \\
\hline
\end{tabular}

Table 1. Total carbon and nitrogen and $\mathrm{KCl}$-extractable ammonium $\left(\mathrm{NH}_{4}{ }^{+}-\mathrm{N}\right)$ and nitrate $\left(\mathrm{NO}_{3}{ }^{-} \mathrm{N}\right)$ in amendments used for the greenhouse trial \pm the standard deviation. ND not determined, as $\mathrm{KCl}$ extraction of slurry is not an appropriate measure plant-available $\mathrm{N}$. Letters indicate significant differences between amendments using a one-way anova $(\mathrm{p}<0.05 ; \mathrm{n}=3)$.

Pyrolysis is one technology which can transform residual biomass into highly porous, surface-functionalized adsorbents $^{22}$. The temperatures used in pyrolysis (typically between 400 and $700{ }^{\circ} \mathrm{C}$ ) assure full sterilization of manure, along with densification and desiccation, leading to safer, and cheaper transportation per unit dry material ${ }^{14,15}$. Much of the literature on biochar sorbents refer to high surface area of low ash biochars ${ }^{23,24}$ derived from plant $\mathrm{s}^{25-29}$. Increasing concern for the environmental burden of manure from animal production has highlighted the relevance of manure biochars as agricultural amendments $\mathrm{s}^{30-33}$. As pyrolysis requires feedstocks to have a moisture content below $15 \%$, a number of strategies have been implemented for reducing the moisture content of manure, such as co-pyrolysis with woody feedstocks, or utilization of the thermochemical byproducts such as emitted heat or bio-oils for drying of moist manures ${ }^{34,35}$.

Manure biochars are notably rich in plant-essential nutrients such as phosphorus and potassium ${ }^{23,36}$, as well as total $\mathrm{N}^{37-39}$. They also have the ability to adsorb residual ammonium $\left(\mathrm{NH}_{4}\right)^{27}$ and volatile ammonia gas $\left(\mathrm{NH}_{3}\right)^{29}$ and can enhance plant $\mathrm{N}$-use efficiency ${ }^{40}$. The ability to sorb $\mathrm{NH}_{3}$ increases after pre-exposure to carbon dioxide $\left(\mathrm{CO}_{2}\right)^{41}$. Since $\mathrm{CO}_{2}$ is a by-product of the pyrolysis process ${ }^{42}$, biochars made from the solid portion of dairy manure can be treated with $\mathrm{CO}_{2}$ before using them to sorb volatile $\mathrm{NH}_{3}$ from slurry lagoons.

Ammonia gas is reported to sorb onto woody biochar and $\mathrm{CO}_{2}$-doped human manure biochar via both strong and weak mechanism ${ }^{27,29,40}$, pointing to both short and long-term plant availability. Sorbed $\mathrm{N}$ from cattle urine onto woody biochar was reported as plant-available, promoting the growth of rye grass ${ }^{43}$. Yet no study has evaluated the actual plant-availability of $\mathrm{NH}_{3}$ sorbed onto manure biochar after exposure to $\mathrm{CO}_{2}$.

While surface charge and acid-base interactions drive $\mathrm{N}$ interactions with biochar made from woody feedstocks, precipitation of $\mathrm{N}$ salts such as ammonium bicarbonate $\left(\mathrm{NH}_{4} \mathrm{HCO}_{3}\right)$ is a more likely mechanism for $\mathrm{N}$ removal by ash-rich dairy manure digestate biochar. Moreover, $\mathrm{NH}_{3}$ loading via $\mathrm{NH}_{4} \mathrm{HCO}_{3}$ precipitation may exceed monolayer surface adsorption to multi-layered sorption through intermittent exposure to $\mathrm{CO}_{2}{ }^{44}$. Recent work demonstrated that exposure of biochar to $\mathrm{NH}_{3}$ re-functionalizes surfaces with amine groups ${ }^{29}$ which are then able to adsorb $\mathrm{CO}_{2}{ }^{41,44-46}$. The incorporation of $\mathrm{CO}_{2}$ molecules may further enhance $\mathrm{NH}_{3}$ retention through the formation of $\mathrm{NH}_{4} \mathrm{HCO}_{3}$, creating $\mathrm{NH}_{4} \mathrm{HCO}_{3}$-intercalated biochar for use as a mineral-organic, slow-release fertilizer. The intimate association between the first layer of chemisorbed $\mathrm{NH}_{3}$ on biochar surfaces and $\mathrm{NH}_{4} \mathrm{HCO}_{3}$ precipitates projecting further out from the surface is expected to provide both long-term and immediately available $\mathrm{N}$. Yet no study has evaluated the plant-availability of $\mathrm{N}$ incorporated into manure biochar through sequential $\mathrm{NH}_{3}$ and $\mathrm{CO}_{2}$ adsorption.

Therefore, we quantify the plant uptake of $\mathrm{N}$ adsorbed to dairy manure biochar using either liquid $\mathrm{NH}_{4}{ }^{+}$ or gaseous $\mathrm{NH}_{3}$ with prior $\mathrm{CO}_{2}$ conditioning. Crops grown in the greenhouse, tomato, marigold, and radish, were used in this small-scale study to demonstrate proof of concept. We benchmarked the performance of dairy manure biochar as an adsorber against wood biochar, a material reported to sorb up to $6 \mathrm{mg} \mathrm{g}^{-1} \mathrm{NH}_{3}-\mathrm{N}^{43,47}$. We compare the plant-availability of $\mathrm{N}$ from biochars exposed to either liquid $\mathrm{NH}_{4}{ }^{+}$or gaseous $\mathrm{NH}_{3}$ to the availability of $\mathrm{N}$ from urea fertilizer added in combination with each biochar. We expect greater plant-availability of $\mathrm{N}$ incorporated to dairy manure biochars compared to that incorporated into wood biochar. We expect greater $\mathrm{N}$ use efficiency from both biochars exposed to $\mathrm{N}$ compared to biochar added with inorganic fertilizer.

\section{Results}

The increase in total $\mathrm{N}$ of the amendments following $\mathrm{NH}_{3}$ exposure was much larger for wood (1.13\% point change in $\mathrm{N}$, from 0.75 to $1.88 \% \mathrm{~N}$ ) than manure biochars (a point change of $0.06 \% \mathrm{~N}$, from 2.05 to $2.11 \% \mathrm{~N}$ ). The increase in $\mathrm{KCl}$-extractable $\mathrm{N}$ ( sum of $\mathrm{NO}_{3}{ }^{-} \mathrm{N}$ and $\mathrm{NH}_{4}{ }^{+}-\mathrm{N}$ ) was similar between wood $\left(0.005-3.1 \mathrm{~g} \mathrm{~N} \mathrm{~kg}^{-1}\right)$ and manure biochars $\left(0.026-3.4 \mathrm{~g} \mathrm{~N} \mathrm{~kg}^{-1}\right)$. The $\mathrm{KCl}$-extractable, or plant-available $\mathrm{N}$ in manure biochar following $\mathrm{NH}_{3}$ exposure increased 127 fold, in comparison to a 595 fold increase for wood biochar, with only $27 \%$ of the added $\mathrm{N}$ in wood biochar following $\mathrm{NH}_{3}$ exposure being plant-available. This increase in plant-available $\mathrm{N}$ versus total $\mathrm{N}$ following $\mathrm{NH}_{3}$ exposure was 21 -fold greater for manure than wood biochar (Table 1). Furthermore, the increase in total $\mathrm{N}$ in manure biochar exposed to $\mathrm{NH}_{3}(0.06 \%$-points), was smaller than the increase in plant-available $\mathrm{N}$ (0.34\%-points) (Table 1$)$. Exposure to $\mathrm{NH}_{3}$ therefore increased the plant-available $\mathrm{N}$ in both biochars to the same extent, apparently, irrespective of the total $\mathrm{N}$ increase.

The change in total $\mathrm{N}$ in manure and wood biochars following immersion in the slurry was similar to that through $\mathrm{NH}_{3}$ exposure, compared to unexposed biochars. However, in both biochars, a much smaller portion of this added $\mathrm{N}$ was immediately plant-available after immersion in slurry, $0.0-0.01 \mathrm{~g} \mathrm{~N} \mathrm{~kg}^{-1}$, compared to after 


\begin{tabular}{|l|l|l|c|c|}
\hline Analysis & Unit & Wood biochar & Manure biochar & Slurry \\
\hline $\mathrm{Al}$ & $\left(\mathrm{g} \mathrm{kg}^{-1}\right)$ & $0.38+0.02 \mathrm{~b}$ & $4.03+1.57 \mathrm{a}$ & $0.35+0.28 \mathrm{~b}$ \\
\hline $\mathrm{Ca}$ & $\left(\mathrm{g} \mathrm{kg}^{-1}\right)$ & $5.3+0.0 \mathrm{~b}$ & $158.1+12.4 \mathrm{a}$ & $49.5+42.0 \mathrm{a}$ \\
\hline $\mathrm{K}$ & $\left(\mathrm{g} \mathrm{kg}^{-1}\right)$ & $5.5+0.3 \mathrm{a}$ & $12.8+1.9 \mathrm{a}$ & $37.5+32.4 \mathrm{a}$ \\
\hline $\mathrm{Mg}$ & $\left(\mathrm{g} \mathrm{kg}^{-1}\right)$ & $0.75+0.01 \mathrm{~b}$ & $15.93+0.12 \mathrm{a}$ & $7.66+6.56 \mathrm{ab}$ \\
\hline $\mathrm{Na}$ & $\left(\mathrm{g} \mathrm{kg}^{-1}\right)$ & $0.47+0.01 \mathrm{a}$ & $3.92+0.51 \mathrm{a}$ & $8.78+7.55 \mathrm{a}$ \\
\hline $\mathrm{P}$ & $\left(\mathrm{g} \mathrm{kg}^{-1}\right)$ & $0.55+0.02 \mathrm{~b}$ & $11.90+0.90 \mathrm{a}$ & $7.17+6.16 \mathrm{ab}$ \\
\hline $\mathrm{S}$ & $\left(\mathrm{g} \mathrm{kg}^{-1}\right)$ & $0.09+0.00 \mathrm{a}$ & $2.30+0.20 \mathrm{a}$ & $3.53+3.03 \mathrm{a}$ \\
\hline Micronutrients $(\mathrm{B}+\mathrm{Cu}+\mathrm{Fe}+\mathrm{Mn}+\mathrm{Zn})$ & $\left(\mathrm{g} \mathrm{kg}^{-1}\right)$ & $0.80+0.03 \mathrm{~b}$ & $3.14+0.19 \mathrm{a}$ & $0.99+0.75 \mathrm{~b}$ \\
\hline Heavy metals $(\mathrm{Cd}, \mathrm{Pb})$ & $\left(\mathrm{mg} \mathrm{kg}^{-1}\right)$ & $3.04+0.33 \mathrm{~b}$ & $15.28+3.64 \mathrm{a}$ & $2.34+1.08 \mathrm{~b}$ \\
\hline
\end{tabular}

Table 2. Total nutrients measured in acid-digested $\left(\mathrm{HClO}_{4}+\mathrm{HNO}_{3}\right)$ amendments used for the greenhouse trial \pm the standard deviation. Letters indicate significant differences between amendments using a one-way anova $(\mathrm{p}<0.05 ; \mathrm{n}=3)$.

\begin{tabular}{|l|l|l|l|l|l|l|l|}
\hline Analysis & Unit & Wood biochar & Manure biochar & $\begin{array}{l}\text { Wood biochar } \\
\mathbf{N H}_{3}+\mathbf{C O}_{2}\end{array}$ & $\begin{array}{l}\text { Manure biochar } \\
\mathbf{C O}_{2}+\mathbf{N H}_{\mathbf{3}}\end{array}$ & Wood biochar + slurry & $\begin{array}{l}\text { Manure } \\
\text { biochar }+ \text { slurry }\end{array}$ \\
\hline $\mathrm{Al}$ & $\left(\mathrm{mg} \mathrm{kg}^{-1}\right)$ & $130.4 \pm 4.6 \mathrm{a}$ & $0.8 \pm 0.5 \mathrm{~d}$ & $83.5 \pm 4.0 \mathrm{~b}$ & $0.0 \pm 0.1 \mathrm{~d}$ & $17.4 \pm 3.6 \mathrm{c}$ & $10.8 \pm 1.6 \mathrm{c}$ \\
\hline $\mathrm{Ca}$ & $\left(\mathrm{g} \mathrm{kg}^{-1}\right)$ & $3.46 \pm 0.06 \mathrm{e}$ & $25.92 \pm 0.03 \mathrm{a}$ & $2.53 \pm 0.08 \mathrm{f}$ & $21.84 \pm 0.16 \mathrm{c}$ & $11.91 \pm 0.36 \mathrm{~d}$ & $23.80 \pm 0.33 \mathrm{~b}$ \\
\hline $\mathrm{K}$ & $\left(\mathrm{g} \mathrm{kg}^{-1}\right)$ & $7.00 \pm 0.05 \mathrm{~d}$ & $9.87 \pm 0.14 \mathrm{c}$ & $6.47 \pm 0.07 \mathrm{~d}$ & $10.41 \pm 0.20 \mathrm{c}$ & $18.70 \pm 0.82 \mathrm{a}$ & $13.12 \pm 0.31 \mathrm{~b}$ \\
\hline $\mathrm{Mg}$ & $\left(\mathrm{g} \mathrm{kg}^{-1}\right)$ & $0.58 \pm 0.02 \mathrm{~d}$ & $3.00 \pm 0.01 \mathrm{a}$ & $0.48 \pm 0.00 \mathrm{~d}$ & $2.50 \pm 0.03 \mathrm{c}$ & $2.46 \pm 0.09 \mathrm{c}$ & $2.71 \pm 0.04 \mathrm{~b}$ \\
\hline $\mathrm{Na}$ & $\left(\mathrm{g} \mathrm{kg}^{-1}\right)$ & $0.81 \pm 0.06 \mathrm{~b}$ & $2.40 \pm 0.01 \mathrm{a}$ & $0.68 \pm 0.04 \mathrm{c}$ & $2.41 \pm 0.00 \mathrm{a}$ & $2.36 \pm 0.00 \mathrm{a}$ & $2.39 \pm 0.00 \mathrm{a}$ \\
\hline $\mathrm{P}$ & $\left(\mathrm{g} \mathrm{kg}^{-1}\right)$ & $0.32 \pm 0.00 \mathrm{~d}$ & $1.08 \pm 0.01 \mathrm{~b}$ & $0.23 \pm 0.00 \mathrm{e}$ & $0.91 \pm 0.00 \mathrm{c}$ & $1.71 \pm 0.06 \mathrm{a}$ & $0.17 \pm 0.03 \mathrm{a}$ \\
\hline $\mathrm{S}$ & $\left(\mathrm{mg} \mathrm{kg}^{-1}\right)$ & $62 \pm 2 \mathrm{c}$ & $302 \pm 4 \mathrm{a}$ & $55 \pm 1 \mathrm{c}$ & $290 \pm 3 \mathrm{a}$ & $214 \pm 9 \mathrm{~b}$ & $303 \pm 8 \mathrm{a}$ \\
\hline $\begin{array}{l}\text { Micronutrients }(\mathrm{B}, \mathrm{Cu}, \\
\text { Fe, Mn, Zn) }\end{array}$ & $\left(\mathrm{mg} \mathrm{kg}^{-1}\right)$ & $358 \pm 9 \mathrm{a}$ & $146 \pm 1 \mathrm{c}$ & $260 \pm 9 \mathrm{~b}$ & $122 \pm 1 \mathrm{~d}$ & $264 \pm 1 \mathrm{~b}$ & $143 \pm 3 \mathrm{c}$ \\
\hline Heavy metals $(\mathrm{Cd}, \mathrm{Pb})$ & $\left(\mathrm{mg} \mathrm{kg}^{-1}\right)$ & $9.83 \pm 3.91 \mathrm{a}$ & $0.84 \pm 0.02 \mathrm{~b}$ & $0.14 \pm 0.32 \mathrm{~b}$ & $0.75 \pm 0.01 \mathrm{~b}$ & $1.42 \pm 0.16 \mathrm{~b}$ & $0.61 \pm 0.06 \mathrm{~b}$ \\
\hline
\end{tabular}

Table 3. Plant-available nutrients in amendments, extracted with Mehlich III \pm the standard deviation. Letters indicate significant differences between amendments using a one-way anova $(\mathrm{p}<0.05 ; \mathrm{n}=3)$.

exposure to $\mathrm{NH}_{3}, 0.005-3.4 \mathrm{~g} \mathrm{~N} \mathrm{~kg}^{-1}$ (Table 1). Thus, sorbed $\mathrm{N}$ on biochars following $\mathrm{NH}_{3}$ exposure is more plant-available than sorbed $\mathrm{N}$ from the slurry.

Manure biochar contained significantly greater total nutrients (acid-digestible) and plant-available (Mehlich III extractable) nutrients by mass than wood biochar, specifically $\mathrm{Ca}, \mathrm{Mg}$, and $\mathrm{P}$ (Tables 2,3 ). This corroborates with previous reports of large amounts of ash minerals in biochars from manure feedstocks compared to woody feedstocks ${ }^{23,36}$. Calcium (Ca) comprised more than $15 \%$ of manure biochar mass and less than $1 \%$ of wood biochar mass. The high $\mathrm{Ca}$ concentration in manure biochar resulted from the regular liming of fresh manure solids after screw-pressing. Significantly greater total micronutrients (B, Cu, Fe, $\mathrm{Mn}, \mathrm{Zn}$ ) were observed in manure biochar compared to wood biochar, 3.14 vs. $0.80 \mathrm{~g} \mathrm{~kg}^{-1}$, respectively. This trend was reversed for extractable elements: wood biochar contained 2.5-fold greater Mehlich III-extractable micronutrients $\left(358.3 \mathrm{mg} \mathrm{g}^{-1}\right)$ than manure biochar $\left(146.1 \mathrm{mg} \mathrm{g}^{-1}\right)$.

The total and Mehlich-III-extractable and therefore plant-available concentrations of heavy metals in both wood and manure biochars were below the EPA threshold values for biosolids intended for agriculture ${ }^{40,48}$. Manure biochar contained significantly greater total concentrations of heavy metals $(\mathrm{Cd}, \mathrm{Pb})$ than wood biochar, 15.3 vs. $3.0 \mathrm{mg} \mathrm{kg}^{-1}$ (Table 2). However, Mehlich III-extractable heavy metals were 12 -fold greater in wood biochar than manure biochar, reaching $9.8 \mathrm{mg} \mathrm{g}^{-1}$ vs. $0.84 \mathrm{mg} \mathrm{g}^{-1}$, respectively (Table 3 ).

Additions of wood biochar alone increased plant growth (dry weight of above and below-ground biomass) from 4.9 to $29 \%$ relative to no additions. Radish plants benefited from additions of manure biochar alone, with $18 \%$ greater plant growth than no additions. When urea alone $(1 \times)$ was added to potting media, plant growth increased by $9-34 \%$ relative to unamended plants. Additions of manure or wood biochar together with urea $(1 \times)$ increased plant growth by $14-63 \%$ relative to unamended plants (Fig. 1, Table 4).

Different forms of $\mathrm{N}$ added with biochars had different effects on plant growth. Wood biochar applied together with urea $(1 \times)$ promoted $0-18 \%$ greater plant growth than wood biochar treated with $\mathrm{NH}_{3}$ despite the fact that the total $\mathrm{N}$ content in both types of amendments was identical, 1.88\%. Furthermore, wood biochar and urea promoted $29-37 \%$ more plant growth compared to slurry-immersed biochar despite the similar total $\mathrm{N}$ values between the two types of amendments, $1.88 \%$ vs $1.84 \%$ (Tables 1,4 ).

Differences in plant growth with the type of biochar and added $\mathrm{N}$ were also apparent. Overall, plant growth was 6-34\% lower after adding manure biochar exposed to $\mathrm{NH}_{3}$ compared to adding wood biochar exposed to $\mathrm{NH}_{3}$. These effects of adding manure biochar exposed to $\mathrm{NH}_{3}$ on plant growth varied between plant type. In a one-way anova of the effect of amendment type on plant growth, between plant types, we observed that 


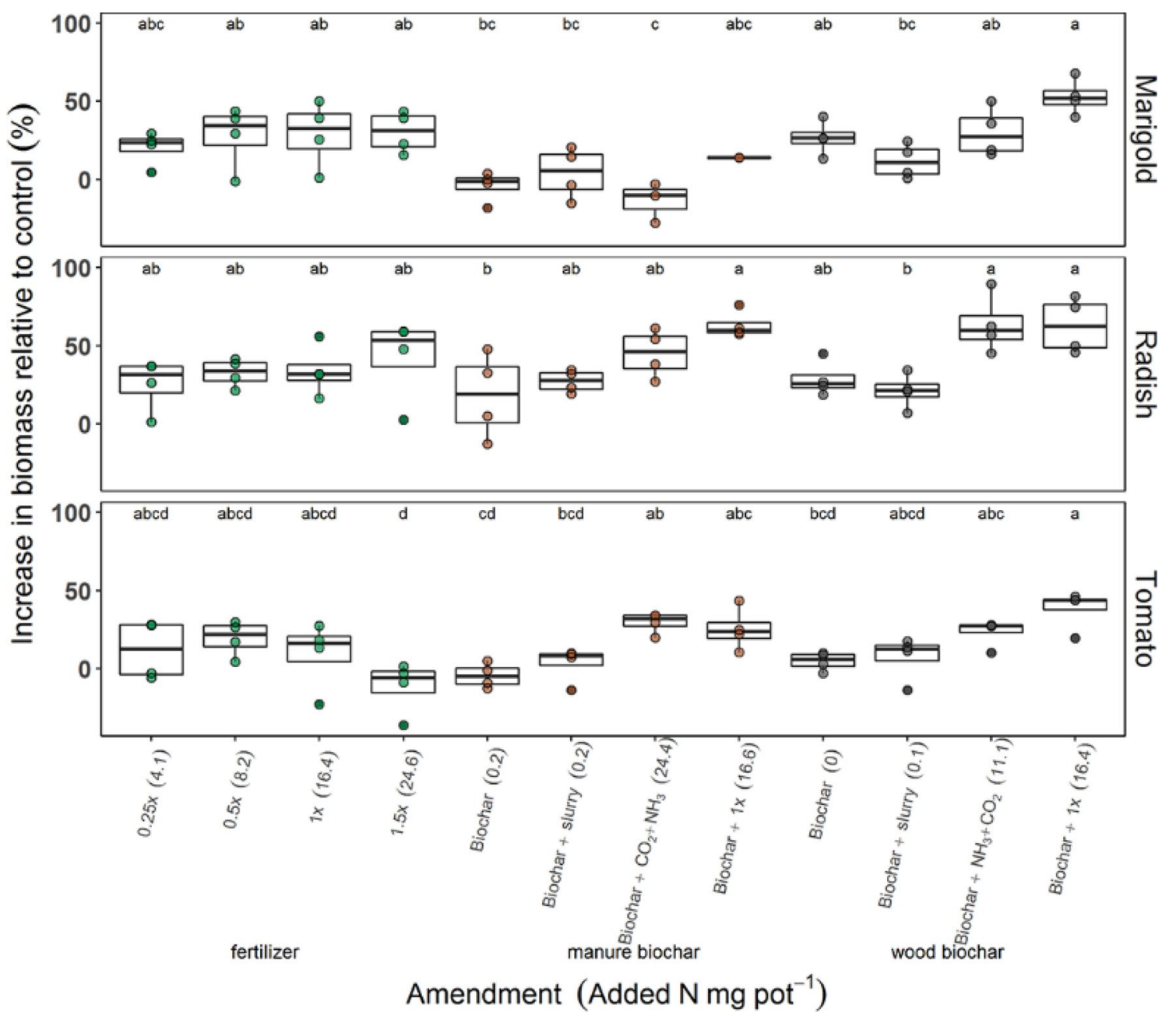

Figure 1. Increase in plant biomass (sum of root and shoot biomass) grown with urea fertilizer (green points), manure biochar (brown points) or wood biochar (gray points) amendments relative to unamended plants $(0 \times)$. The amount of plant-available $\mathrm{N}$ in each type of amendment in parentheses. Letters above the bars indicate significant differences between amendments within plant type $(p<0.05 ; n=4$; whiskers indicate standard errors).

supplementing manure biochar with $\mathrm{N}$ by exposing it to $\mathrm{NH}_{3}$ significantly improved tomato growth above tomatoes grown with only manure biochar, by $35 \%$. Supplementing manure biochar with urea fertilizer significantly improved radish growth by $38 \%$ compared to additions of manure biochar alone (Fig. 1, Table 4).

Using a two-way anova of the effect of amendments and plant types on growth, plant growth in radishes amended with manure or wood biochar added with urea fertilizer and wood biochar treated with $\mathrm{NH}_{3}$ were significantly higher compared to the growth of all plant types grown with manure biochar alone. Furthermore, no significant differences in plant growth were apparent between radish and tomato amended with manure or wood biochars treated with $\mathrm{NH}_{3}$ or added with urea fertilizer, compared to adding urea alone, in any amount (Fig. SI 1, Table SI 5). Thus, supplementing wood or manure biochar with $\mathrm{N}$ from $\mathrm{NH}_{3}$ exposure or urea fertilizer proved just as good for plants as adding urea fertilizer alone.

Germination was affected by plant type and amendment, and was lower with greater nutrient additions for marigold and radish, and higher with the highest nutrient additions for tomato. Unamended and manure biocharamended marigold and radish had the highest germination rates, $80-90 \%$, while tomato plants amended with the highest urea application rates, $1.5 \times$, and wood biochar, reached $100 \%$ germination (Table SI 4 ).

After 40 days, the $\mathrm{pH}$ of pots amended with manure biochar (7.02-7.46) was significantly higher than the potting mix amended with urea $(1 \times)$ or the unamended potting mix (6.11-6.48), based on both a one-way anova of the effect of amendment type on $\mathrm{pH}$ (Table 4) and a two-way anova of the effect of amendment type and plant type on $\mathrm{pH}$ (Table SI 5). The $\mathrm{pH}$ of pots with wood biochar immersed in the liquid manure (6.47-7.03) was highest among all wood biochar treatments (6.25-6.74). In contrast, wood biochar alone did not significantly increase the $\mathrm{pH}$ of the potting mix, relative to the unamended pots (Table 4, Table SI 5).

Plant $\mathrm{N}$ uptake increased with increasing $\mathrm{N}$ additions (Fig. 2). The range of urea additions encompassed $\mathrm{N}$ additions of all other amendments (Fig. 3) indicating positive growth responses across all rates of added $\mathrm{N}$. Nitrogen from wood biochar exposed to $\mathrm{NH}_{3}$ appears to be as plant available as urea $1 \times$, since $\mathrm{N}$ uptake was slightly higher per unit $\mathrm{N}$ added (Fig. 3). Furthermore, the $\mathrm{N}$ uptake of plants grown with wood biochar treated with $\mathrm{NH}_{3}$ was not significantly different than the $\mathrm{N}$ uptake of plants grown with urea $1 \times$, based on either the 


\begin{tabular}{|c|c|c|c|c|c|c|}
\hline Plant & Amendment & pH after 40 days & Plant $\mathrm{N}$ uptake $\left(\mathrm{mg} \mathrm{pot}^{-1}\right)$ & $\begin{array}{l}\text { Relative increase in plant } \\
\mathrm{N} \text { uptake }\left(\% \mathrm{w} \mathrm{w}^{-1}\right)\end{array}$ & Plant biomass $\left(\mathrm{g} \mathrm{pot}^{-1}\right)$ & $\begin{array}{l}\text { Relative increase in plant } \\
\text { biomass }\left(\% \mathrm{w} \mathrm{w}^{-1}\right)\end{array}$ \\
\hline Marigold & $0 \times$ fert & $6.35 \pm 0.13 \mathrm{~cd}$ & $35.63 \pm 3.87 \mathrm{de}$ & NA & $2.81 \pm 0.36 \mathrm{bc}$ & NA \\
\hline Marigold & $0.25 \times$ fert & $6.28 \pm 0.14 \mathrm{~cd}$ & $40.37 \pm 7.21 \mathrm{bcde}$ & $13.28 \pm 20.25 \mathrm{bcde}$ & $3.38 \pm 0.31 \mathrm{abc}$ & $20.27 \pm 10.89 \mathrm{abc}$ \\
\hline Marigold & $0.5 \times$ fert & $6.30 \pm 0.04 \mathrm{~cd}$ & $38.97 \pm 6.45 \mathrm{cde}$ & $9.36 \pm 18.10 \mathrm{cde}$ & $3.59 \pm 0.57 \mathrm{ab}$ & $27.73 \pm 20.15 \mathrm{ab}$ \\
\hline Marigold & $1 \times$ fert & $6.15 \pm 0.14 \mathrm{~d}$ & $51.63 \pm 4.49 \mathrm{abc}$ & $44.89 \pm 12.61 \mathrm{abc}$ & $3.63 \pm 0.60 \mathrm{ab}$ & $28.98 \pm 21.20 \mathrm{ab}$ \\
\hline Marigold & $1.5 \times$ fert & $6.25 \pm 0.11 \mathrm{~cd}$ & $61.36 \pm 6.64 \mathrm{a}$ & $72.20 \pm 18.65 \mathrm{a}$ & $3.66 \pm 0.37 \mathrm{ab}$ & $30.22 \pm 13.25 \mathrm{ab}$ \\
\hline Marigold & Manure biochar & $7.46 \pm 0.16 \mathrm{a}$ & $31.50 \pm 5.24 \mathrm{e}$ & $-11.60 \pm 14.71 \mathrm{e}$ & $2.69 \pm 0.27 b c$ & $-4.27 \pm 9.67 \mathrm{bc}$ \\
\hline Marigold & Manure biochar + slurry & $7.35 \pm 0.43 \mathrm{a}$ & $34.10 \pm 4.93 \mathrm{de}$ & $-4.30 \pm 13.83 \mathrm{de}$ & $2.93 \pm 0.46 \mathrm{bc}$ & $4.00 \pm 16.51 \mathrm{bc}$ \\
\hline Marigold & Manure biochar $\mathrm{CO}_{2}+\mathrm{NH}_{3}$ & $7.41 \pm 0.24 \mathrm{a}$ & $42.74 \pm 4.38 \mathrm{bcde}$ & $19.95 \pm 12.29 \mathrm{bcde}$ & $2.43 \pm 0.36 \mathrm{c}$ & $-13.72 \pm 12.77 \mathrm{c}$ \\
\hline Marigold & Manure biochar $+1 \times$ & $7.35 \pm 0.04 \mathrm{a}$ & $52.28 \pm 0.00 \mathrm{abc}$ & $46.71 \pm 0.00 \mathrm{abc}$ & $3.20 \pm 0.00 \mathrm{abc}$ & $13.78 \pm 0.00 \mathrm{abc}$ \\
\hline Marigold & Wood biochar & $6.53 \pm 0.19 \mathrm{~cd}$ & $32.40 \pm 2.46 \mathrm{e}$ & $-9.08 \pm 6.92 \mathrm{e}$ & $3.56 \pm 0.31 \mathrm{ab}$ & $26.58 \pm 10.89 \mathrm{ab}$ \\
\hline Marigold & Wood biochar + slurry & $7.03 \pm 0.18 \mathrm{ab}$ & $33.20 \pm 1.63 \mathrm{de}$ & $-6.84 \pm 4.56 \mathrm{de}$ & $3.14 \pm 0.31 \mathrm{bc}$ & $11.64 \pm 11.16 \mathrm{bc}$ \\
\hline Marigold & Wood biochar $\mathrm{NH}_{3}+\mathrm{CO}_{2}$ & $6.62 \pm 0.13 \mathrm{bc}$ & $46.44 \pm 9.15 \mathrm{bcd}$ & $30.33 \pm 25.67 \mathrm{bcd}$ & $3.66 \pm 0.44 \mathrm{ab}$ & $30.22 \pm 15.82 \mathrm{ab}$ \\
\hline Marigold & Wood biochar $+1 \times$ & $6.51 \pm 0.15 \mathrm{~cd}$ & $53.33 \pm 3.45 \mathrm{ab}$ & $49.66 \pm 9.67 \mathrm{ab}$ & $4.30 \pm 0.33 \mathrm{a}$ & $52.80 \pm 11.58 \mathrm{a}$ \\
\hline Radish & $0 \times$ fert & $6.48 \pm 0.12 \mathrm{~cd}$ & $29.60 \pm 7.33 \mathrm{~d}$ & NA & $3.08 \pm 0.27 \mathrm{c}$ & NA \\
\hline Radish & $0.25 \times$ fert & $6.51 \pm 0.16 \mathrm{~cd}$ & $40.06 \pm 4.41 \mathrm{bcd}$ & $35.33 \pm 14.89 \mathrm{bcd}$ & $3.87 \pm 0.52 \mathrm{abc}$ & $25.39 \pm 16.91 \mathrm{ab}$ \\
\hline Radish & $0.5 \times$ fert & $6.34 \pm 0.14 \mathrm{~d}$ & $42.74 \pm 7.71 \mathrm{abcd}$ & $44.37 \pm 26.04 \mathrm{abcd}$ & $4.09 \pm 0.28 \mathrm{abc}$ & $32.69 \pm 9.13 \mathrm{ab}$ \\
\hline Radish & $1 \times$ fert & $6.35 \pm 0.15 \mathrm{~d}$ & $53.04 \pm 6.73 \mathrm{ab}$ & $79.17 \pm 22.74 \mathrm{ab}$ & $4.13 \pm 0.50 \mathrm{abc}$ & $34.06 \pm 16.35 \mathrm{ab}$ \\
\hline Radish & $1.5 \times$ fert & $6.37 \pm 0.14 \mathrm{~d}$ & $55.94 \pm 8.38 \mathrm{a}$ & $88.96 \pm 28.30 \mathrm{a}$ & $4.39 \pm 0.83 \mathrm{ab}$ & $42.25 \pm 26.80 \mathrm{ab}$ \\
\hline Radish & Manure biochar & $7.22 \pm 0.10 \mathrm{ab}$ & $30.19 \pm 4.82 \mathrm{~d}$ & $1.99 \pm 16.27 \mathrm{~d}$ & $3.65 \pm 0.84 \mathrm{bc}$ & $18.25 \pm 27.22 b$ \\
\hline Radish & Manure biochar + slurry & $7.31 \pm 0.12 \mathrm{a}$ & $31.60 \pm 1.78 \mathrm{~d}$ & $6.74 \pm 6.00 \mathrm{~d}$ & $3.93 \pm 0.22 \mathrm{abc}$ & $27.33 \pm 7.19 \mathrm{ab}$ \\
\hline Radish & Manure biochar $\mathrm{CO}_{2}+\mathrm{NH}_{3}$ & $7.25 \pm 0.13 a$ & $55.28 \pm 8.68 \mathrm{a}$ & $86.75 \pm 29.34 \mathrm{a}$ & $4.48 \pm 0.48 \mathrm{ab}$ & $45.26 \pm 15.45 \mathrm{ab}$ \\
\hline Radish & Manure biochar $+1 \times$ & $7.02 \pm 0.56 \mathrm{ab}$ & $46.72 \pm 1.03 \mathrm{abc}$ & $57.82 \pm 3.49 \mathrm{abc}$ & $5.04 \pm 0.27 \mathrm{a}$ & $63.42 \pm 8.62 \mathrm{a}$ \\
\hline Radish & Wood biochar & $6.74 \pm 0.04 \mathrm{bcd}$ & $31.68 \pm 3.45 \mathrm{~d}$ & $7.01 \pm 11.64 \mathrm{~d}$ & $3.97 \pm 0.35 \mathrm{abc}$ & $28.79 \pm 11.34 \mathrm{ab}$ \\
\hline Radish & Wood biochar + slurry & $6.90 \pm 0.16 \mathrm{abc}$ & $32.58 \pm 2.55 \mathrm{~cd}$ & $10.05 \pm 8.61 \mathrm{~cd}$ & $3.74 \pm 0.35 \mathrm{bc}$ & $21.17 \pm 11.28 \mathrm{~b}$ \\
\hline Radish & Wood biochar $\mathrm{NH}_{3}+\mathrm{CO}_{2}$ & $6.45 \pm 0.06 \mathrm{~cd}$ & $50.52 \pm 8.56 \mathrm{ab}$ & $70.65 \pm 28.92 \mathrm{ab}$ & $5.05 \pm 0.58 \mathrm{a}$ & $63.67 \pm 18.83 \mathrm{a}$ \\
\hline Radish & Wood biochar $+1 \times$ & $6.48 \pm 0.14 \mathrm{~cd}$ & $48.51 \pm 2.66 \mathrm{ab}$ & $63.88 \pm 8.97 \mathrm{ab}$ & $5.03 \pm 0.55 a$ & $63.10 \pm 17.80 \mathrm{a}$ \\
\hline Tomato & $0 \times$ fert & $6.11 \pm 0.07 \mathrm{~d}$ & $39.70 \pm 3.52 \mathrm{def}$ & NA & $3.52 \pm 0.43 \mathrm{bcd}$ & NA \\
\hline Tomato & $0.25 \times$ fert & $6.15 \pm 0.18 \mathrm{~d}$ & $46.36 \pm 4.73 \mathrm{cde}$ & $16.79 \pm 11.92 \mathrm{cde}$ & $3.93 \pm 0.66 \mathrm{abcd}$ & $11.81 \pm 18.76 \mathrm{abcd}$ \\
\hline Tomato & $0.5 \times$ fert & $6.33 \pm 0.55 \mathrm{~cd}$ & $52.98 \pm 2.68 \mathrm{bc}$ & $33.46 \pm 6.74 \mathrm{bc}$ & $4.20 \pm 0.40 \mathrm{abcd}$ & $19.49 \pm 11.37 \mathrm{abcd}$ \\
\hline Tomato & $1 \times$ fert & $6.17 \pm 0.02 \mathrm{~d}$ & $60.72 \pm 3.40 \mathrm{ab}$ & $52.95 \pm 8.58 \mathrm{ab}$ & $3.84 \pm 0.78 \mathrm{abcd}$ & $9.10 \pm 22.11 \mathrm{abcd}$ \\
\hline Tomato & $1.5 \times$ fert & $6.36 \pm 0.30 \mathrm{~cd}$ & $71.55 \pm 11.10 \mathrm{a}$ & $80.23 \pm 27.97 \mathrm{a}$ & $3.11 \pm 0.60 \mathrm{~d}$ & $-11.59 \pm 16.98 \mathrm{~d}$ \\
\hline Tomato & Manure biochar & $7.08 \pm 0.12 \mathrm{a}$ & $34.26 \pm 2.42 \mathrm{f}$ & $-13.69 \pm 6.09 f$ & $3.36 \pm 0.28 \mathrm{~cd}$ & $-4.48 \pm 7.89 \mathrm{~cd}$ \\
\hline Tomato & Manure biochar + slurry & $7.07 \pm 0.13 \mathrm{a}$ & $36.95 \pm 1.50 \mathrm{ef}$ & $-6.91 \pm 3.78 \mathrm{ef}$ & $3.63 \pm 0.40 \mathrm{bcd}$ & $3.13 \pm 11.34 \mathrm{bcd}$ \\
\hline Tomato & Manure biochar $\mathrm{CO}_{2}+\mathrm{NH}_{3}$ & $6.83 \pm 0.46 \mathrm{abc}$ & $51.64 \pm 4.60 \mathrm{bc}$ & $30.09 \pm 11.58 \mathrm{bc}$ & $4.55 \pm 0.24 \mathrm{ab}$ & $29.30 \pm 6.71 \mathrm{ab}$ \\
\hline Tomato & Manure biochar $+1 \times$ & $7.05 \pm 0.03 \mathrm{ab}$ & $49.75 \pm 3.39 \mathrm{bcd}$ & $25.32 \pm 8.55 \mathrm{bcd}$ & $4.40 \pm 0.48 \mathrm{abc}$ & $25.18 \pm 13.67 \mathrm{abc}$ \\
\hline Tomato & Wood biochar & $6.30 \pm 0.14 \mathrm{~cd}$ & $35.60 \pm 3.47 \mathrm{ef}$ & $-10.33 \pm 8.74 \mathrm{ef}$ & $3.68 \pm 0.21 \mathrm{bcd}$ & $4.62 \pm 5.89 \mathrm{bcd}$ \\
\hline Tomato & Wood biochar + slurry & $6.47 \pm 0.12 \mathrm{bcd}$ & $40.16 \pm 2.27 \mathrm{def}$ & $1.16 \pm 5.73 \mathrm{def}$ & $3.77 \pm 0.50 \mathrm{abcd}$ & $7.18 \pm 14.22 \mathrm{abcd}$ \\
\hline Tomato & Wood biochar $\mathrm{NH}_{3}+\mathrm{CO}_{2}$ & $6.32 \pm 0.11 \mathrm{~cd}$ & $51.79 \pm 1.76 \mathrm{bc}$ & $30.45 \pm 4.42 \mathrm{bc}$ & $4.33 \pm 0.31 \mathrm{abc}$ & $23.19 \pm 8.73 \mathrm{abc}$ \\
\hline Tomato & Wood biochar $+1 \times$ & $6.25 \pm 0.05 \mathrm{~cd}$ & $57.20 \pm 4.41 \mathrm{bc}$ & $44.10 \pm 11.11 \mathrm{bc}$ & $4.86 \pm 0.44 \mathrm{a}$ & $38.26 \pm 12.56 \mathrm{a}$ \\
\hline Marigold & $0 \times$ fert & $6.35 \pm 0.13 \mathrm{~cd}$ [ghij] & $35.63 \pm 3.87 \mathrm{de}$ [ghijklm] & NA & $2.81 \pm 0.36 \mathrm{bc}[\mathrm{hij}]$ & NA \\
\hline Marigold & $0.25 \times$ fert & $6.28 \pm 0.14 \mathrm{~cd}[\mathrm{hij}]$ & $\begin{array}{l}40.37 \pm 7.21 \text { bcde [efghi- } \\
\text { jklm] }\end{array}$ & $13.28 \pm 20.25 \mathrm{bcde}$ [ghijklm] & $3.38 \pm 0.31 \mathrm{abc}$ [defghij] & $20.27 \pm 10.89 \mathrm{abc}[\mathrm{bcdefg}]$ \\
\hline Marigold & $0.5 \times$ fert & $6.30 \pm 0.04 \mathrm{~cd}[\mathrm{hij}]$ & $38.97 \pm 6.45$ cde [efghijklm] & $9.36 \pm 18.10$ cde [ghijklm] & $3.59 \pm 0.57 \mathrm{ab}[\mathrm{cdefghij}]$ & $27.73 \pm 20.15 \mathrm{ab}[\mathrm{abcdefg}]$ \\
\hline Marigold & $1 \times$ fert & $6.15 \pm 0.14 \mathrm{~d}[\mathrm{j}]$ & $51.63 \pm 4.49 \mathrm{abc}[\mathrm{bcdef}]$ & $44.89 \pm 12.61 \mathrm{abc}$ [bcdefghi] & $3.63 \pm 0.60 \mathrm{ab}$ [cdefghij] & $28.98 \pm 21.20 \mathrm{ab}[\mathrm{abcdefg}]$ \\
\hline Marigold & $1.5 \times$ fert & $6.25 \pm 0.11 \mathrm{~cd}[\mathrm{hij}]$ & $61.36 \pm 6.64 \mathrm{a}[\mathrm{ab}]$ & $72.20 \pm 18.65 \mathrm{a}[\mathrm{abcd}]$ & $3.66 \pm 0.37 \mathrm{ab}$ [cdefghij] & $30.22 \pm 13.25 \mathrm{ab}$ [abcdef] \\
\hline Marigold & Manure biochar & $7.46 \pm 0.16 \mathrm{a}[]$ & $31.50 \pm 5.24 \mathrm{e}[\mathrm{m}]$ & $-11.60 \pm 14.71 \mathrm{e}[\mathrm{lm}]$ & $2.69 \pm 0.27 \mathrm{bc}[\mathrm{ij}]$ & $-4.27 \pm 9.67 \mathrm{bc}[\mathrm{efg}]$ \\
\hline Marigold & Manure biochar + slurry & $7.35 \pm 0.43 \mathrm{a}[]$ & $34.10 \pm 4.93 \mathrm{de}[\mathrm{ijklm}]$ & $-4.30 \pm 13.83 \mathrm{de}[\mathrm{jklm}]$ & $2.93 \pm 0.46 \mathrm{bc}$ [ghij] & $4.00 \pm 16.51 \mathrm{bc}[\mathrm{cdefg}]$ \\
\hline Marigold & Manure biochar $\mathrm{CO}_{2}+\mathrm{NH}_{3}$ & $7.41 \pm 0.24 \mathrm{a}[]$ & \begin{tabular}{|l}
$42.74 \pm 4.38$ bcde [defghi- \\
jklm]
\end{tabular} & $\begin{array}{l}\text { 19.95 } \pm 12.29 \text { bcde [efghi- } \\
\text { jklm] }\end{array}$ & $2.43 \pm 0.36 \mathrm{c}[\mathrm{j}]$ & $-13.72 \pm 12.77 \mathrm{c}[\mathrm{fg}]$ \\
\hline Marigold & Manure biochar $+1 \times$ & $7.35 \pm 0.04 \mathrm{a}[]$ & $52.28 \pm 0.00 \mathrm{abc}[\mathrm{bcdefgh}]$ & $46.71 \pm 0.00 \mathrm{abc}$ [abcdefghij] & $3.20 \pm 0.00 \mathrm{abc}[\mathrm{defghij}]$ & $13.78 \pm 0.00 \mathrm{abc}$ [abcdefg] \\
\hline Marigold & Wood biochar & $6.53 \pm 0.19 \mathrm{~cd}[]$ & $32.40 \pm 2.46 \mathrm{e}[\mathrm{jklm}]$ & $-9.08 \pm 6.92 \mathrm{e}[\mathrm{klm}]$ & $3.56 \pm 0.31 \mathrm{ab}$ [defghij] & $26.58 \pm 10.89 \mathrm{ab}[\mathrm{abcdefg}]$ \\
\hline Marigold & Wood biochar + slurry & $7.03 \pm 0.18 \mathrm{ab}[]$ & $33.20 \pm 1.63 \mathrm{de}[\mathrm{jklm}]$ & $-6.84 \pm 4.56 \mathrm{de}[\mathrm{jklm}]$ & $3.14 \pm 0.31 \mathrm{bc}$ [efghij] & $11.64 \pm 11.16 \mathrm{bc}[\mathrm{bcdefg}]$ \\
\hline Marigold & Wood biochar $\mathrm{NH}_{3}+\mathrm{CO}_{2}$ & $6.62 \pm 0.13 \mathrm{bc}[]$ & $46.44 \pm 9.15$ bcd [cdefghijk] & $30.33 \pm 25.67 \mathrm{bcd}$ [defghijkl] & $3.66 \pm 0.44 \mathrm{ab}$ [cdefghij] & $30.22 \pm 15.82 \mathrm{ab}$ [abcdef] \\
\hline Marigold & Wood biochar $+1 \times$ & $6.51 \pm 0.15 \mathrm{~cd}[]$ & $53.33 \pm 3.45 \mathrm{ab}[\mathrm{bcde}]$ & $49.66 \pm 9.67 \mathrm{ab}$ [abcdefgh] & $4.30 \pm 0.33 \mathrm{a}$ [abcdef] & $52.80 \pm 11.58 \mathrm{a}[\mathrm{ab}]$ \\
\hline Radish & $0 \times$ fert & $6.48 \pm 0.12 \mathrm{~cd}[]$ & $29.60 \pm 7.33 \mathrm{~d}[\mathrm{~m}]$ & NA & $3.08 \pm 0.27 \mathrm{c}[\mathrm{fghij}]$ & NA \\
\hline Radish & $0.25 \times$ fert & $6.51 \pm 0.16 \mathrm{~cd}[]$ & $40.06 \pm 4.41 \mathrm{bcd}$ [efghijklm] & $35.33 \pm 14.89 \mathrm{bcd}$ [cdefghij] & $3.87 \pm 0.52 \mathrm{abc}$ [abcdefghi] & $25.39 \pm 16.91 \mathrm{ab}[\mathrm{abcdefg}]$ \\
\hline
\end{tabular}




\begin{tabular}{|c|c|c|c|c|c|c|}
\hline Plant & Amendment & $\mathrm{pH}$ after $\mathbf{4 0}$ days & Plant N uptake $\left(\mathrm{mg} \mathrm{pot}^{-1}\right)$ & $\begin{array}{l}\text { Relative increase in plant } \\
\mathrm{N} \text { uptake }\left(\% \mathrm{w} \mathrm{w}^{-1}\right)\end{array}$ & Plant biomass ( g pot $^{-1}$ ) & $\begin{array}{l}\text { Relative increase in plant } \\
\text { biomass }\left(\% \mathrm{w} \mathrm{w}^{-1}\right)\end{array}$ \\
\hline Radish & $0.5 \times$ fert & $6.34 \pm 0.14 \mathrm{~d}[]$ & $\begin{array}{l}42.74 \pm 7.71 \mathrm{abcd} \text { [defghi- } \\
\text { jklm] }\end{array}$ & $\begin{array}{l}\begin{array}{l}44.37 \pm 26.04 a b c d ~[b c d e f- \\
\text { ghi] }\end{array}\end{array}$ & $4.09 \pm 0.28 \mathrm{abc}[\mathrm{abcdefgh}]$ & $32.69 \pm 9.13 \mathrm{ab}[\mathrm{abcde}]$ \\
\hline Radish & $1 \times$ fert & $6.35 \pm 0.15 \mathrm{~d}[]$ & $53.04 \pm 6.73 \mathrm{ab}[\mathrm{bcde}]$ & $79.17 \pm 22.74 \mathrm{ab}[\mathrm{abc}]$ & $4.13 \pm 0.50 \mathrm{abc}$ [abcdefg] & $34.06 \pm 16.35 \mathrm{ab}$ [abcde] \\
\hline Radish & $1.5 \times$ fert & $6.37 \pm 0.14 \mathrm{~d}[]$ & $55.94 \pm 8.38 \mathrm{a}[\mathrm{bcd}]$ & $88.96 \pm 28.30 \mathrm{a}[\mathrm{a}]$ & $4.39 \pm 0.83 \mathrm{ab}$ [abcde] & $42.25 \pm 26.80 \mathrm{ab}$ [abcd] \\
\hline Radish & Manure biochar & $7.22 \pm 0.10 \mathrm{ab}[]$ & $30.19 \pm 4.82 \mathrm{~d}[\mathrm{~m}]$ & $1.99 \pm 16.27 \mathrm{~d}[\mathrm{ijklm}]$ & $3.65 \pm 0.84 \mathrm{bc}$ [cdefghij] & $18.25 \pm 27.22 \mathrm{~b}[\mathrm{bcdefg}]$ \\
\hline Radish & Manure biochar + slurry & $7.31 \pm 0.12 \mathrm{a}[]$ & $31.60 \pm 1.78 \mathrm{~d}[\mathrm{~lm}]$ & $6.74 \pm 6.00 \mathrm{~d}[\mathrm{hijklm}]$ & $3.93 \pm 0.22 \mathrm{abc}$ [abcdefghi] & $27.33 \pm 7.19 \mathrm{ab}$ [abcdefg] \\
\hline Radish & Manure biochar $\mathrm{CO}_{2}+\mathrm{NH}_{3}$ & $7.25 \pm 0.13 \mathrm{a}[]$ & $55.28 \pm 8.68 \mathrm{a}[\mathrm{bcd}]$ & $86.75 \pm 29.34 \mathrm{a}[\mathrm{ab}]$ & $4.48 \pm 0.48 \mathrm{ab}[\mathrm{abcd}]$ & $45.26 \pm 15.45 \mathrm{ab}[\mathrm{abc}]$ \\
\hline Radish & Manure biochar $+1 \times$ & $7.02 \pm 0.56 \mathrm{ab}[]$ & $46.72 \pm 1.03 \mathrm{abc}$ [bcdefghij] & $57.82 \pm 3.49 \mathrm{abc}$ [abcdef] & $5.04 \pm 0.27 \mathrm{a}[\mathrm{a}]$ & $63.42 \pm 8.62 \mathrm{a}[\mathrm{a}]$ \\
\hline Radish & Wood biochar & $6.74 \pm 0.04 \mathrm{bcd}[]$ & $31.68 \pm 3.45 \mathrm{~d}[\mathrm{klm}]$ & $7.01 \pm 11.64 \mathrm{~d}$ [hijklm] & $3.97 \pm 0.35 \mathrm{abc}$ [abcdefghi] & $28.79 \pm 11.34 \mathrm{ab}[\mathrm{abcdefg}]$ \\
\hline Radish & Wood biochar + slurry & $6.90 \pm 0.16 \mathrm{abc}[]$ & $32.58 \pm 2.55 \mathrm{~cd}[\mathrm{jklm}]$ & $10.05 \pm 8.61 \mathrm{~cd}$ [ghijklm] & $3.74 \pm 0.35 \mathrm{bc}[\mathrm{bcdefghij}]$ & $21.17 \pm 11.28 \mathrm{~b}[\mathrm{bcdefg}]$ \\
\hline Radish & Wood biochar $\mathrm{NH}_{3}+\mathrm{CO}_{2}$ & $6.45 \pm 0.06 \mathrm{~cd}[]$ & $50.52 \pm 8.56 \mathrm{ab}[\mathrm{bcdef}]$ & $70.65 \pm 28.92 \mathrm{ab}[\mathrm{abcd}]$ & $5.05 \pm 0.58 \mathrm{a}[\mathrm{a}]$ & $63.67 \pm 18.83 \mathrm{a}[\mathrm{a}]$ \\
\hline Radish & Wood biochar $+1 \times$ & $6.48 \pm 0.14 \mathrm{~cd}[]$ & $48.51 \pm 2.66 \mathrm{ab}[\mathrm{bcdefghi}]$ & $63.88 \pm 8.97 \mathrm{ab}$ [abcde] & $5.03 \pm 0.55 \mathrm{a}[\mathrm{ab}]$ & $63.10 \pm 17.80 \mathrm{a}[\mathrm{a}]$ \\
\hline Tomato & $0 \times$ fert & $6.11 \pm 0.07 \mathrm{~d}[]$ & $39.70 \pm 3.52$ def [efghijklm] & NA & $3.52 \pm 0.43 \mathrm{bcd}$ [defghij] & NA \\
\hline Tomato & $0.25 \times$ fert & $6.15 \pm 0.18 \mathrm{~d}[]$ & $46.36 \pm 4.73$ cde [cdefghijkl] & $16.79 \pm 11.92$ cde [fghijklm] & $3.93 \pm 0.66 \mathrm{abcd}$ [abcdefghi] & $11.81 \pm 18.76 \mathrm{abcd}$ [bcdefg] \\
\hline Tomato & $0.5 \times$ fert & $6.33 \pm 0.55 \mathrm{~cd}[]$ & $52.98 \pm 2.68 \mathrm{bc}[\mathrm{bcde}]$ & $33.46 \pm 6.74 \mathrm{bc}$ [defghijk] & $4.20 \pm 0.40 \mathrm{abcd}[\mathrm{abcdefg}]$ & $19.49 \pm 11.37 \mathrm{abcd}$ [bcdefg] \\
\hline Tomato & $1 \times$ fert & $6.17 \pm 0.02 \mathrm{~d}[]$ & $60.72 \pm 3.40 \mathrm{ab}[\mathrm{abc}]$ & $52.95 \pm 8.58 \mathrm{ab}$ [abcdefg] & $3.84 \pm 0.78 \mathrm{abcd}$ [abcdefghi] & $9.10 \pm 22.11 \mathrm{abcd}[\mathrm{cdefg}]$ \\
\hline Tomato & $1.5 \times$ fert & $6.36 \pm 0.30 \mathrm{~cd}[]$ & $71.55 \pm 11.10 \mathrm{a}[\mathrm{a}]$ & $80.23 \pm 27.97 \mathrm{a}[\mathrm{ab}]$ & $3.11 \pm 0.60 \mathrm{~d}[\mathrm{efghij}]$ & $-11.59 \pm 16.98 \mathrm{~d}[\mathrm{~g}]$ \\
\hline Tomato & Manure biochar & $7.08 \pm 0.12 \mathrm{a}[]$ & $34.26 \pm 2.42 \mathrm{f}[\mathrm{hijklm}]$ & $-13.69 \pm 6.09 f[\mathrm{~m}]$ & $3.36 \pm 0.28 \mathrm{~cd}$ [defghij] & $-4.48 \pm 7.89 \mathrm{~cd}[\mathrm{efg}]$ \\
\hline Tomato & Manure biochar + slurry & $7.07 \pm 0.13 \mathrm{a}[]$ & $36.95 \pm 1.50$ ef [fghijklm] & $-6.91 \pm 3.78 \mathrm{ef}[\mathrm{jklm}]$ & $3.63 \pm 0.40 \mathrm{bcd}$ [cdefghij] & $3.13 \pm 11.34 \mathrm{bcd}[\mathrm{defg}]$ \\
\hline Tomato & Manure biochar $\mathrm{CO}_{2}+\mathrm{NH}_{3}$ & $6.83 \pm 0.46 \mathrm{abc}[]$ & $51.64 \pm 4.60 \mathrm{bc}[\mathrm{bcdef}]$ & $\begin{array}{l}30.09 \pm 11.58 \mathrm{bc} \text { [defghi- } \\
\text { jklm] }\end{array}$ & $4.55 \pm 0.24 \mathrm{ab}[\mathrm{abcd}]$ & $29.30 \pm 6.71 \mathrm{ab}$ [abcdefg] \\
\hline Tomato & Manure biochar $+1 \times$ & $7.05 \pm 0.03 \mathrm{ab}[]$ & $49.75 \pm 3.39 \mathrm{bcd}$ [bcdefg] & $25.32 \pm 8.55 \mathrm{bcd}$ [efghijklm] & $4.40 \pm 0.48 \mathrm{abc}$ [abcde] & $25.18 \pm 13.67 \mathrm{abc}$ [abcdefg] \\
\hline Tomato & Wood biochar & $6.30 \pm 0.14 \mathrm{~cd}[]$ & $35.60 \pm 3.47 \mathrm{ef}$ [ghijklm] & $-10.33 \pm 8.74 \mathrm{ef}[\mathrm{klm}]$ & $3.68 \pm 0.21 \mathrm{bcd}$ [cdefghij] & $4.62 \pm 5.89 \mathrm{bcd}[\mathrm{cdefg}]$ \\
\hline Tomato & Wood biochar + slurry & $6.47 \pm 0.12 \mathrm{bcd}[]$ & $40.16 \pm 2.27 \mathrm{def}[$ efghijklm] & $1.16 \pm 5.73 \mathrm{def}[\mathrm{ijklm}]$ & $\begin{array}{l}3.77 \pm 0.50 \text { abcd [abcdef- } \\
\text { ghij] }\end{array}$ & $7.18 \pm 14.22 \mathrm{abcd}[\mathrm{cdefg}]$ \\
\hline Tomato & Wood biochar $\mathrm{NH}_{3}+\mathrm{CO}_{2}$ & $6.32 \pm 0.11 \mathrm{~cd}[]$ & $51.79 \pm 1.76 \mathrm{bc}[\mathrm{bcde}]$ & $30.45 \pm 4.42 \mathrm{bc}$ [defghijkl] & $4.33 \pm 0.31 \mathrm{abc}$ [abcdef] & $23.19 \pm 8.73 \mathrm{abc}[\mathrm{abcdefg}]$ \\
\hline Tomato & Wood biochar $+1 \times$ & $6.25 \pm 0.05 \mathrm{~cd}[]$ & $57.20 \pm 4.41 \mathrm{bc}[\mathrm{abcd}]$ & $44.10 \pm 11.11 \mathrm{bc}$ [bcdefghi] & $4.86 \pm 0.44 \mathrm{a}[\mathrm{abc}]$ & $38.26 \pm 12.56 \mathrm{a}[\mathrm{abcd}]$ \\
\hline
\end{tabular}

Table 4. The average \pm standard deviation of the $\mathrm{pH}$ of potting mix and amendments after 40 days, total plant biomass (shoot and root combined) and relative increase in plant biomass relative to unamended plants $(0 \times)$, total $\mathrm{N}$ plant uptake (shoot and root combined), and relative increase in biomass $\mathrm{N}$ uptake in comparison to unamended plants. Letters indicate significant differences from a one-way anova between amendments within plant type ( $\mathrm{p}<0.05 ; \mathrm{n}=4$; results for a two-way anova include in supplementary online material Table SI 5).

one-way anova of the effect of amendment on $\mathrm{N}$ uptake, between plant types or the two-way anova evaluating $\mathrm{N}$ uptake as affected by both amendment and plant type (Fig. 2, Table 4, Fig. SI 2, Table SI 5). Nitrogen from manure biochar exposed to $\mathrm{NH}_{3}$ appears to be less plant available than urea, since $\mathrm{N}$ uptake was smaller per unit $\mathrm{N}$ added (Fig. 3), although these results varied between plant type. Radish plants grown with manure biochar treated with $\mathrm{NH}_{3}$ had similar $\mathrm{N}$ uptake as when grown with the highest urea treatment.

When added with urea, biochar did not increase $\mathrm{N}$ uptake, compared to $\mathrm{N}$ uptake with urea additions alone. However, adsorption of $\mathrm{NH}_{3}$ onto biochars provided both immediately-available plant-available $\mathrm{N}$ and longer-term mineralizable $\mathrm{N}$, allowing for greater plant uptake than equivalent amendments of urea. The initial KCl-extractable $\mathrm{N}$ in manure biochar exposed to $\mathrm{NH}_{3}$ was comparable to urea $0.5 \times$ additions, indicating the mineralizability of $\mathrm{N}$ incorporated through $\mathrm{NH}_{3}$-exposure of manure biochar (Fig. 3). The difference in total $\mathrm{N}$ in wood biochar following $\mathrm{NH}_{3}$ exposure was equal to the amount of $\mathrm{N}$ in urea $1 \times$ addition, yet the KClextractable $\mathrm{N}$ was comparable to the amount of $\mathrm{N}$ in urea $0.25 \times$. Nevertheless, compared to urea $0.25 \times$, plant $\mathrm{N}$ uptake in wood biochar sorbed with $\mathrm{NH}_{3}$ was $12-26 \%$ greater. Moreover, in all experiments, $\mathrm{NH}_{3}$-exposed biochars outperformed biochars immersed in the liquid fraction, increasing $\mathrm{N}$ uptake by $29-55 \%$ for wood biochar and $25-75 \%$ for manure.

\section{Discussion}

The feedstock and pyrolysis conditions used to produce biochar for these experiments did not result in phototoxicity, as no difference in germination was detectable between plants with or without biochar. The moderately high temperatures used for pyrolysis, $500{ }^{\circ} \mathrm{C}$ may have reduced the phototoxicity reported from lower-temperature biochars ${ }^{49}$. The greater increase in $\mathrm{KCl}$-extractable $\mathrm{N}$ than in total $\mathrm{N}$ in manure biochar exposed to $\mathrm{NH}_{3}$ may be related to $\mathrm{CO}_{2}$ exposure that increased extractability of the $\mathrm{N}$ already present in the biochar. Yet it is unclear whether $\mathrm{CO}_{2}$ can solubilize trapped inorganic $\mathrm{N}$ species or cleave bonds of organic $\mathrm{N}$. Either way, all $\mathrm{N}$ from $\mathrm{NH}_{3}$ added to manure biochar was $\mathrm{KCl}$-extractable. On the other hand, the increase in total $\mathrm{N}$ in wood biochar exposed to $\mathrm{NH}_{3}$ was 19 -fold greater than manure biochar exposed to $\mathrm{NH}_{3}$, even though both materials contained the same amount of $\mathrm{KCl}$-extractable $\mathrm{N}$. This means that the majority of $\mathrm{N}$ in wood biochar treated with $\mathrm{NH}_{3}$ more strongly interacted with the biochar than the $\mathrm{NH}_{3}$-derived $\mathrm{N}$ in manure biochar, in alignment with previous studies ${ }^{29,40}$ and was therefore not plant-available. 


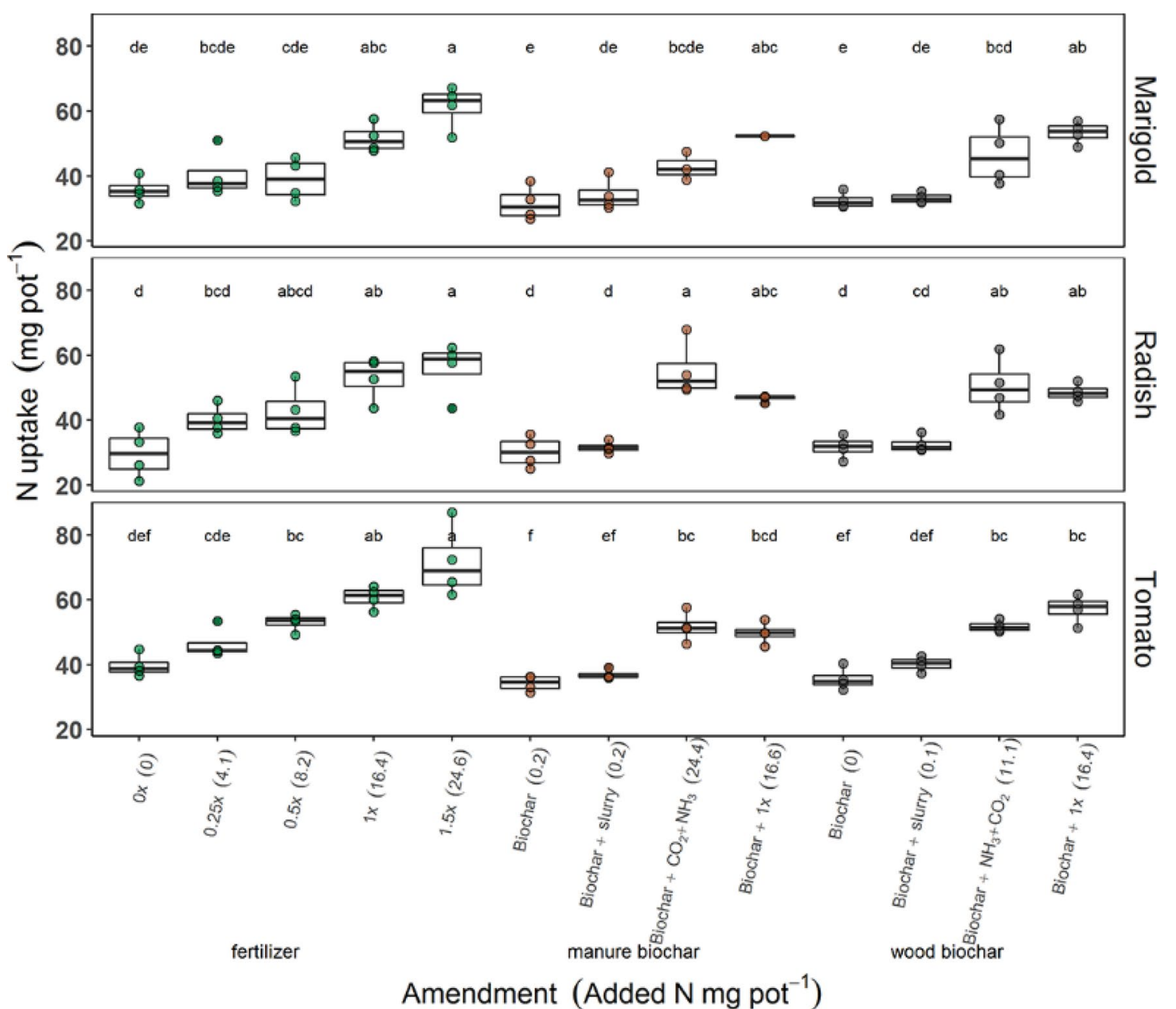

Figure 2. Total nitrogen uptake in shoot and root biomass of plants grown with urea fertilizer, manure biochar or wood biochar amendments or no amendments $(0 \times)$. Letters above the bars indicate significant differences between amendments within plant type $(p<0.05 ; \mathrm{n}=4)$.

Capturing volatile $\mathrm{NH}_{3}$ from the liquid fraction of dairy waste onto sorbents such as biochars was more successful for recycling nutrients for use as potting media than immersing sorbents in the liquid fraction. Plant $\mathrm{N}$ uptake was $25-75 \%$ and $29-55 \%$ greater in manure and wood biochars, respectively, for the $\mathrm{NH}_{3}$ treatment compared to slurry immersion. Lower plant growth and $\mathrm{N}$ uptake with biochar treated with the slurry could be attributed to a combination of both the lower amount of readily plant-available $\mathrm{N}$ (i.e., KCl-extractable) and lower rates of mineralization of organic $\mathrm{N}$. The plant-availability of organic $\mathrm{N}$ in the liquid fraction is time-dependent and varies with manure age and processing. Pettygrove et al. ${ }^{50}$ determined that $27-44 \%$ of total $\mathrm{N}$ in fresh lagoon slurry was mineralized after 6 weeks when incubated in a sandy loam soil. The low $\mathrm{pH}$ of our peat potting media may have slowed microbial mineralization rates of slurry $\mathrm{N}$ compared to rates reported for mineral soils ${ }^{51}$.

Delivering $\mathrm{N}$ from $\mathrm{NH}_{3}$-enriched manure biochar to plants was just as effective as adding urea with manure biochar. The increase in plant $\mathrm{N}$ uptake relative to the control was $43-55 \%$ vs. $47-52 \%$, respectively. The high plant-availability of $\mathrm{N}$ in manure biochar doped with $\mathrm{N}$ may indicate that an $\mathrm{NH}_{4}{ }^{+}$salt such as $\mathrm{NH}_{4} \mathrm{HCO}_{3}$ formed from repeated exposure to $\mathrm{NH}_{3}$ and $\mathrm{CO}_{2}$, preserving all sorbed $\mathrm{N}$ in a plant-available form, as observed with $\mathrm{CO}_{2}$ captured within $\mathrm{NH}_{3}$ solvents ${ }^{52-54}$. Mineral forms of $\mathrm{N}$ were detected in human manure biochars and wood biochars exposed to repeated intervals of $\mathrm{NH}_{3}$ and $\mathrm{CO}_{2}$ using XPS, although two-fold more electrostaticallysorbed $\mathrm{NH}_{4}{ }^{+}$was detected in $\mathrm{NH}_{3}$ - and $\mathrm{CO}_{2}$-exposed wood biochar compared to $\mathrm{NH}_{3}$ - and $\mathrm{CO}_{2}$-exposed manure biochar $^{40}$.

Using median international prices for fertilizer nutrients, the value of extractable $\mathrm{N}$ in biochars exposed to $\mathrm{NH}_{3}$ is $1.7 \mathrm{USD} \mathrm{Mg}^{-1}$ for manure biochar and $1.5 \mathrm{USD} \mathrm{Mg}^{-1}$ for wood biochar ${ }^{39}$. The benefit of plant-extractable minerals (Mehlich-III extractable) in dairy manure biochar on plant growth was not evaluated experimentally, since equivalent extractable nutrients in manure biochar were added to all other treatments. Nevertheless, we can estimate the added monetary value of plant-extractable $\mathrm{P}$ and $\mathrm{K}$ in manure biochar, together with plant-

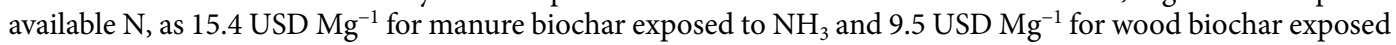
to $\mathrm{NH}_{3}$ using literature values ${ }^{39}$.

The increase in plant $\mathrm{N}$ uptake with additions of manure biochar exposed to $\mathrm{NH}_{3}$ relative to unamended plants reached $20-87 \%$ or $7.1-25.7 \mathrm{mg} \mathrm{N} \mathrm{pot}^{-1}$, which, accounting for the amount of biochar added to each pot, 


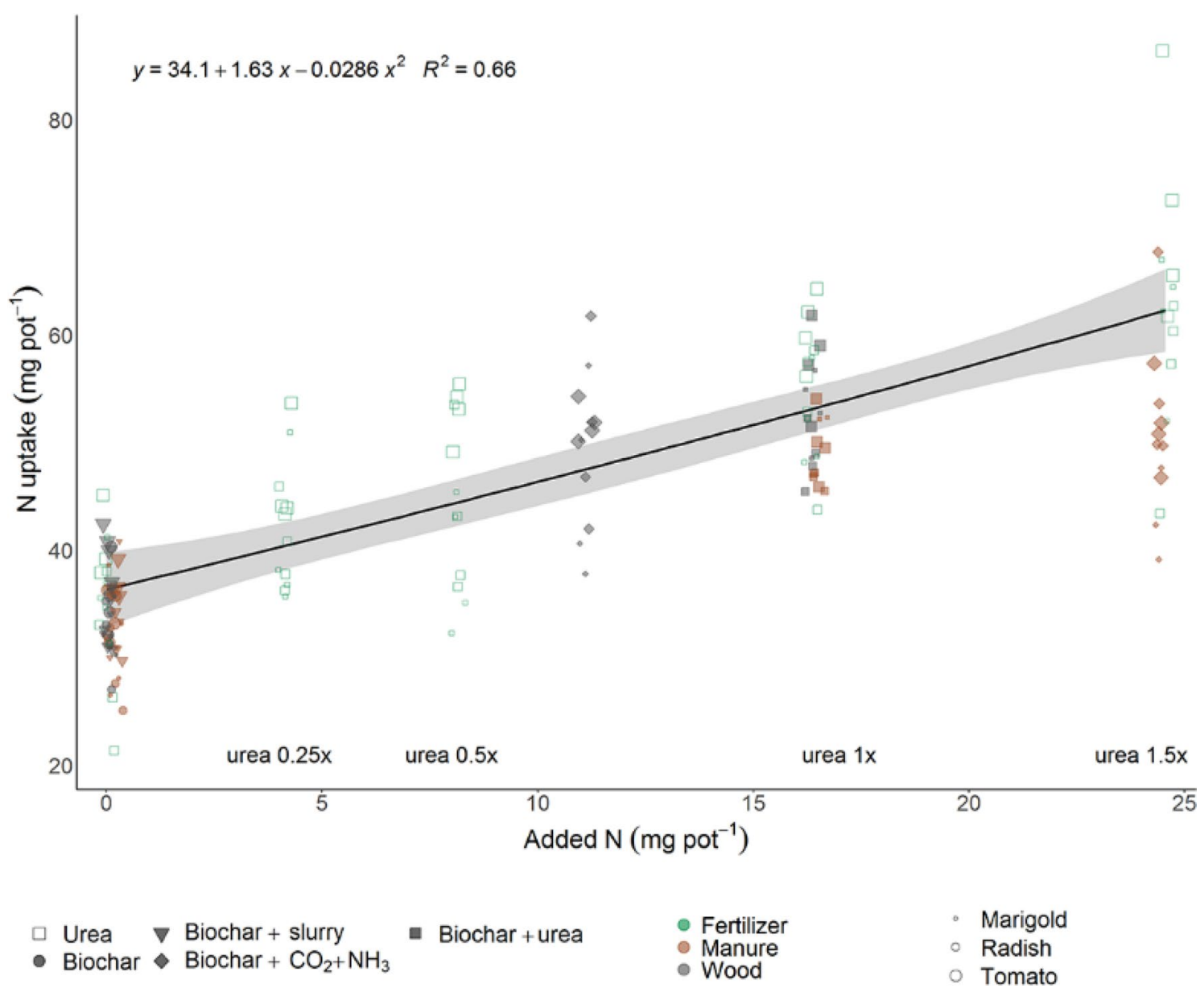

Figure 3. Nitrogen uptake of shoots and roots of plants grown with amendments varying in initial $\mathrm{N}$ content (points). "Added N" refers to the $\mathrm{KCl}$-extractable $\mathrm{N}$ of all amendments, with the assumption that all urea-N is $\mathrm{KCl}$-extractable. A linear regression was conducted for urea-based amendments (urea, biochar + urea). The 95\% confidence interval is shown by the gray shaded line, and the $\mathrm{R}^{2}$ value for the quadratic equation is presented $(n=4)$.

is equivalent to $1-3.6 \mathrm{~kg} \mathrm{~N} \mathrm{Mg}^{-1}$ manure biochar. If we account for more than 624,000 dairy cows in NY State $\mathrm{e}^{55}$, each generating approximately $18.8 \mathrm{Mg}$ (dry) manure year ${ }^{-156}$, this amount of available $\mathrm{N}_{\text {in }} \mathrm{NH}_{3}$-exposed manure biochar scales to $11,732-42,232 \mathrm{Mg} \mathrm{N}_{\text {year }}{ }^{-1}$ or 6-21.5 million USD year ${ }^{-1}$, based on the average price of $\mathrm{N}$ fertilizer $\left(0.51 \mathrm{USD} \mathrm{kg}^{-139}\right)$. With approximately $51,360 \mathrm{Mg} \mathrm{N}$ applied to grain corn in NY State in $2018^{4,6}$, separated dairy manure treated with $\mathrm{NH}_{3}$ can offset $23-82 \%$ of $\mathrm{N}$ fertilizer needs while stabilizing both the solid and liquid fraction of manure for addressing both environmental pollution as well as recycling $\mathrm{N}$ to agriculture.

A novel fertilizer has been developed from $\mathrm{N}$-rich dairy waste which performs equally well compared to conventional urea fertilizer per unit applied $\mathrm{N}$. Not only did $\mathrm{NH}_{3}$-sorbed wood biochar contain similar amounts of plant-available $\mathrm{N}$ as $\mathrm{NH}_{3}$-manure biochar, but promoted greater plant biomass growth and plant- $\mathrm{N}$ uptake than manure biochar or conventional urea fertilizer. Despite the greater $\mathrm{NH}_{3}$-derived $\mathrm{N}$ enrichment in wood than manure biochar, the precipitation of $\mathrm{NH}_{3}$-salts on manure biochar in comparison to chemisorption of $\mathrm{NH}_{3}$ to wood, points at manure biochar being a more efficient approach to recycle manure $\mathrm{N}$ as a fertilizer.

This research demonstrates that it is possible to convert dairy manure solids into a biochar that can adsorb volatile $\mathrm{NH}_{3}$ for use as a $\mathrm{N}$ fertilizer. Conversion of dairy manures with high water contents into a dry and $\mathrm{N}$-rich soil amendment with $\mathrm{N}$ use efficiency by plants commensurate with urea fertilizer may provide life-cycle benefits to water quality and greenhouse gas emissions that should be studied in the future. Future studies should include scaling up $\mathrm{CO}_{2}$ and $\mathrm{NH}_{3}$ exposure of biochar. Multi-year field studies with crops such as corn should examine the long-term availability of $\mathrm{N}$-doped biochars and potential differences in leaching and gaseous $\mathrm{N}$ losses. The feasibility of cost-effectively operating dairy manure pyrolysis as well as adsorption of $\mathrm{N}$ to biochars should be studied on farms and by small industry. Techno-economic studies should quantify the ability to optimize the production and distribution of such fertilizers at different scales and under different economic conditions.

\section{Materials and methods}

Enriching biochar amendments with nitrogen. We evaluated the effect of N-enriched dairy manure biochar and $\mathrm{N}$-enriched wood biochar on plant growth. The first type of biochar was created from anaerobicallydigested dairy manure solids ('solid fraction'), screw-pressed at a dairy farm in upstate New York in April 2018. 
The solid fraction was charred in a modified muffle furnace with a rotating paddle at $500{ }^{\circ} \mathrm{C}$ for $30 \mathrm{~min}$. The liquid fraction of the screw-pressed dairy manure was also collected, sieved with a $425 \mu \mathrm{m}$ mesh sieve to remove solids, and stored at $-4{ }^{\circ} \mathrm{C}$. The second type of biochar made from Douglas fir wood (Pseudotsuga menziesii) using high-temperature gasification, was provided by Green Tree Garden Supply (Ithaca, NY). Both biochars were sieved to below $2 \mathrm{~mm}$ particle size.

Manure biochar and wood biochar were enriched with $\mathrm{N}$ through two methods: (1) repeated, sequential exposure to $\mathrm{CO}_{2}$ and $\mathrm{NH}_{3}$, or (2) immersion in the sieved liquid fraction ('liquid fraction'), which contained a mixture of $\mathrm{N}$ species. The effect of these two $\mathrm{N}$-enriched amendments on plant growth was compared to (3) separate additions of each biochar (manure or wood biochar) with urea fertilizer, (4) urea additions without biochar, (5) separate additions of each biochar without urea fertilizer, or (6) no additions of biochar or urea.

For the first $\mathrm{N}$-enrichment method, $200 \mathrm{~g}$ of biochar were loaded in a 4-L Buechner funnel suspended upright inside a drying oven at $30^{\circ} \mathrm{C}$. The bottom of the funnel was connected to gas flow via silicone tubing, and the top covered with a lid and with parafilm. Manure biochar was first exposed to $\mathrm{CO}_{2}$ gas (Instrument grade, Airgas, Ithaca, NY) for one hour. After one hour, $\mathrm{CO}_{2}$ flow ceased, and $\mathrm{NH}_{3}$ flow commenced for one hour. Ammonia gas was generated by pumping air at $4.72 \times 10^{-4} \mathrm{~m}^{3} \mathrm{~s}^{-1}$ through a sealed Erlenmeyer flask containing $1 \mathrm{~L}$ of $2 \mathrm{M} \mathrm{NH}_{4} \mathrm{OH}\left(\mathrm{pH} 12.43\right.$ ) kept on a hot plate at $30^{\circ} \mathrm{C}$. This process was repeated three times (manure biochar $\mathrm{CO}_{2}+\mathrm{NH}_{3}$ ). To enrich wood biochar with $\mathrm{N}$, we reversed the order of gas exposure, first $\mathrm{NH}_{3}$ then $\mathrm{CO}_{2}$, also for three exposure intervals (wood biochar $\mathrm{NH}_{3}+\mathrm{CO}_{2}$ ).

For the second $\mathrm{N}$-enrichment method, biochars were immersed in the liquid fraction for $1.5 \mathrm{~h}$ at a ratio of $143 \mathrm{~g}: 1 \mathrm{~L}$. The biochar-slurry suspension was contained in a large glass beaker on a hot plate at $30^{\circ} \mathrm{C}$. After the immersion period, the suspension was sieved through a $425-\mu \mathrm{m}$ mesh sieve to remove residual liquid. Biochars were dried at $80^{\circ} \mathrm{C}$ for 2 days. We did not expose slurry-treated biochars to $\mathrm{CO}_{2}$.

Duplicate sets of each biochar- $\mathrm{N}$ mixture were homogenized and stored in sealed glass jars. The four biochar treatments evaluated were: (1) manure biochar treated with $\mathrm{CO}_{2}+\mathrm{NH}_{3}$; (2) wood biochar treated with $\mathrm{NH}_{3}+\mathrm{CO}_{2}$; (3) dairy manure biochar immersed in the liquid fraction; and (4) wood biochar immersed in the liquid fraction. To simplify notation, when describing biochars treated with $\mathrm{CO}_{2}+\mathrm{NH}_{3}$ (manure) or $\mathrm{NH}_{3}+\mathrm{CO}_{2}$ (wood), we will refer to $\mathrm{NH}_{3}$ exposure without referring to $\mathrm{CO}_{2}$, as $\mathrm{NH}_{3}$ uptake was our focus.

Chemical analysis of amendments. Subsamples of amendments were milled and analyzed for total C and $\mathrm{N}$ by dry combustion (Elementar; vario EL cube, Langenselbold, Germany). Non-milled amendment subsamples were extracted with $2 \mathrm{M} \mathrm{KCl}$ at a ratio of $0.1 \mathrm{~g}$ biochar $\mathrm{mL}^{-1} \mathrm{KCl}$ and tested for $\mathrm{NH}_{4}^{+}$and $\mathrm{NO}_{3}{ }^{-}$through a colorimetric method on an auto-flow analyzer (AA3 HR AutoAnalyzer, Seal Analytical, Mequon, WI).

Non-milled amendment samples were also analyzed for plant-available and total elements. Plant-available nutrients were extracted using a Mehlich III solution at a ratio of $0.1 \mathrm{~g} \mathrm{~mL}^{-1}$. Total elemental analysis was conducted on $0.5 \mathrm{~g}$ of unmilled biochar spiked with $0.25 \mathrm{mg} \mathrm{L}^{-1}$ yttrium as an internal standard. Samples were dissolved in a mixture of $30 \%$ perchloric acid in nitric acid $(70 \%)$ at $180^{\circ} \mathrm{C}$. Both Mehlich III extracts and digestate solutions were analyzed by inductively-coupled plasma optical emission spectroscopy (ICP-OES; Spectro Arcos, Ametek Materials Analysis, Kleve, Germany).

Greenhouse trial to evaluate amendments. A six-week growth trial was conducted to test amendment performance. Three types of plants, either marigold, radish, and tomato, were grown in a peat potting mix (TH6, Theriault and Hachey Peat Moss Ltd., Baie Sainte-Anne, New Brunswick Canada) in square pots $0.3 \mathrm{~L}$ by volume to which biochars were added. Seeds were obtained from commercial companies (radish and tomato from Burpee and marigold from Park Seed) and its use complies with relevant institutional, national, and international guidelines and legislation. Wood biochar amendments were added at $10 \%$ bulk volume of the square pots, while manure biochar amendments were added at an equivalent $\mathrm{C}$ amount as wood biochar. This resulted in unequal mass and nutrients additions (other than $\mathrm{C}$ ) between wood biochar and manure biochar. To correct for the increased non- $\mathrm{N}$ nutrient addition from manure biochar a mixture of dry nutrients was added to all non-manure biochar treatments based on the Mehlich III extractable nutrient content of manure biochar (Tables SI 1, Table SI 2).

We tested the effect of urea additions to compare the effect of $\mathrm{N}$ source on plant growth (Table SI 2). Nitrogen sources included biochars enriched in $\mathrm{N}$ through exposure to $\mathrm{NH}_{3}$, biochars enriched in $\mathrm{N}$ through immersion into the liquid fraction, and urea fertilizer additions. Urea additions were adjusted to equal the increase in total $\mathrm{N}$ on wood biochar after sequential exposure to $\mathrm{NH}_{3}$ and $\mathrm{CO}_{2}$ (Table SI 3), $4.9 \mathrm{~g} \mathrm{~N} \mathrm{~kg}$ biochar ${ }^{-1}$. We did not add slurry alone, as we focused on adding nutrients and fertilizers as dry materials.

For each plant type, five seeds were planted in each pot after filling with the respective media and nutrient additions. Seedlings were thinned to a single seedling after two weeks, and the germination rate recorded for each pot. Pots in which no seeds germinated received seedlings from replicate pots of the same treatment in which more than one seed germinated.

The $\mathrm{N}$ equivalency of the $\mathrm{N}$ added with the biochar was quantified in comparison to an $\mathrm{N}$-response curve measured from plants grown with urea fertilizer but without biochar (Urea 99\% reagent grade, Sigma Aldrich). Five urea application rates were tested based on the increase in total $\mathrm{N}$ on wood biochar $+\mathrm{NH}_{3}$ (Table 1, Table SI 3): (1) 1.5 times the $\mathrm{N}$ increase of wood biochar after $\mathrm{NH}_{3}$ and $\mathrm{CO}_{2}$ exposure $(1.5 \times)=52.65 \mathrm{mg} \mathrm{N}^{-1}$ or $176.8 \mathrm{~kg} \mathrm{~N} \mathrm{ha}^{-1}$, (2) the equivalent $\mathrm{N}$-application rate as the $\mathrm{N}$ increase of wood biochar $(1 \times)=35.10 \mathrm{mg} \mathrm{N}^{-1}$ or $117.9 \mathrm{~kg} \mathrm{~N} \mathrm{ha}^{-1}$, (3) half of the $\mathrm{N}$ increase $(0.5 \times)=17.55 \mathrm{mg} \mathrm{N} \mathrm{pot}^{-1}$ or $58.95 \mathrm{~kg} \mathrm{~N} \mathrm{ha}^{-1}$, (4) one quarter of the $\mathrm{N}$ increase $(0.25 \times)=8.78 \mathrm{mg} \mathrm{N}^{-1}$ or $29.47 \mathrm{~kg} \mathrm{~N} \mathrm{ha}^{-1}$, and (5) no added fertilizer $(0 \times)$. The urea $1 \times$ addition is in the range of the suggested $\mathrm{N}$ application for corn production in New York State $\left(78-146 \mathrm{~kg} \mathrm{~N} \mathrm{ha}^{-1}\right.$ without 
sod history, legume mixture or manure additions), which is highly variable with soil type and with cropping history ${ }^{57}$. As mentioned above, we also tested the plant effect of each biochar added with urea (1×) (Table SI 3).

All plants were irrigated daily with reverse osmosis water to $90 \%$ of field capacity, determined gravimetrically. The field capacity of TH6 media amended with wood biochar or manure biochar was calculated as the amount of water remaining in a $0.3 \mathrm{~L}$ PVC cylinder filled with soil after saturation and draining overnight. Irrigation was lowered from field capacity by $10 \%$ to prevent leaching during the experiment.

To overcome the hydrophobicity of the potting mixtures, pots were initially placed in trays of water to moisten them from the bottom-up. From the second day of the experiment until day 12, pots were misted from the top daily. After germination on day 12, pots were weighed to determine the amount of water needed to reach $90 \%$ of field capacity. A description of the thirteen potting mixtures and irrigation amounts is provided in the supplementary information (Table SI 2).

Plants were harvested after 40 days, and wet shoot and root biomass recorded. Shoots were cut at the soil surface, and roots were excavated from each pot. Roots were isolated via washing and sieving. Dry root and shoot biomass was determined after drying at $65^{\circ} \mathrm{C}$ for 3 days. The potting mix was dried at $105{ }^{\circ} \mathrm{C}$ for 3 days. Total $\mathrm{C}$ and $\mathrm{N}$ contents in shoots and roots were determined by dry combustion. After the experiment, all potting mixtures were extracted with Mehlich III to determine changes in plant-available nutrients. The pH of potting mixtures was measured before and after the growth trial in $1 \mathrm{~g}$ potting mixture in $20 \mathrm{~mL}$ deionized water.

For each of three plant types (marigold $=i_{1}$, tomato $=i_{2}$, radish $=i_{3}$ ) and biomass type (roots $=j_{1}$, shoots $=j_{2}$ ), the proportional increase in biomass and $\mathrm{N}$ uptake across four replicates $(k=1: 4)$ of amended plants relative to the average of four replicates of unamended plants for the same plant and biomass type was calculated as follows:

$$
\begin{aligned}
& \text { Proportion of biomass }\left(N \text { uptake) } \text { increase }_{i, j, k}\right. \\
& =\frac{\text { biomass }\left(\mathrm{N} \text { uptake) amended plants }{ }_{i . j . k}-\text { biomass }(N \text { uptake }) \text { unamended plants } s_{i, j, k}\right.}{\text { biomass }(N \text { uptake }) \text { unamended plants } s_{i, j}} \times 100(\%)
\end{aligned}
$$

The N-fertilizer equivalency of amendments was calculated based on the difference in $\mathrm{N}$ uptake of plants grown with urea alone $(1 \times)$ and plants grown with biochar amendments. Using market prices for mineral fertilizers $^{39}$, we also calculated the replacement value of the plant-available $\mathrm{N}, \mathrm{P}$, and $\mathrm{K}$ in amendments.

Statistics. Data analysis was carried out in RStudio ${ }^{58}$ and graphs created using ggplot $2^{59}$. Least squares of treatment means (LS means) were calculated using emmeans ${ }^{60}$. Order-independent $p$ values determined with the Student- $t$ test were adjusted using Tukey's method for comparing a family of five estimates at the $\alpha=0.05$ threshold. Compact letter displays of pairwise comparisons for a significance level of $p<0.05$ were created using multcompView ${ }^{61}$. Type I analysis of variance (ANOVA) was calculated using an order-dependent $F$ test within the emmeans package. We present a one-way anova of the effect of amendment types on plant growth (dry weight) and plant $\mathrm{N}$ uptake in the main manuscript, and include both one-way anova and two-way anova evaluating the effect of amendment type and plant type, and the interaction between them, on plant growth (dry weight) and $\mathrm{N}$ uptake in the supplementary material. All mention of 'significant differences' refers to the probability of observing an $F$ ratio greater than 0.05 given the null hypothesis, $\operatorname{Pr}(>F)$, or a $p$ value $<0.05$.

Received: 25 February 2021; Accepted: 5 July 2021

Published online: 22 July 2021

\section{References}

1. NYSDAM (New York State Department of Agriculture and Markets) New York State Dairy Statistics 2017 annual summary. NYS Agricultural Statistics Service. https://agriculture.ny.gov/system/files/documents/2019/06/NYSAnnStat2017.pdf (Department of Agriculture and Markets, Division of Milk Control and DairyServices, 2017).

2. Lander, C. H., Moffitt, D. \& Alt, K. Nutrients available from livestock manure relative to crop growth requirements; Appendix II- manure characteristics. https://www.nrcs.usda.gov/wps/portal/nrcs/detail/national/technical/nra/nri/results/?cid=nrcs143_ 014175 (United States Department of Agriculture (USDA) Natural Resource Conservation Service (NRCS), 1998).

3. Magdoff, F. R. \& van Es, H. M. (eds.) Building soils for better crops: Sustainable soilmanagement, 3rd edn. Handbook Series 10. (Sustainable Agriculture Research and Extension, College Park, MD, 2009).

4. Mosheim, R. Fertilizer Use and Price (USDA Economic Research Service, 2019).

5. Wightman, J. L. \& Woodbury, P. B. New York dairy manure management greenhouse gas emissions and mitigation costs (19922022). J. Environ. Qual. 45, 266-275 (2016).

6. USDA NASS (US Department of Agriculture, National Agricultural StatisticsService). 2014-2015 Agricultural Statistics Annual Bulletin, New York. https://www.nass.usda.gov/Statistics_by_State/New_York/Publications/Annual_Statistical_Bulletin/2015/ 2014-2015\%20NY\%20Annual\%20Bulletin.pdf (New York Agricultural Statistics USDA, National Agricultural Statistics Service, 2014).

7. Scharf, P. C., Kitchen, N. R., Sudduth, K. A. \& Davis, J. G. Spatially variable corn yield is a weak predictor of optimal nitrogen rate. Soil Sci. Soc. Am. J. 70, 2154-2160 (2006).

8. Shepherd, T. Karszes, J. \& Gooch, C. Covered manure storage cost calculator. http://northeast.manuremanagement.cornell.edu/ Pages/Assessment_Tools/Covered_Storage_Calculator.html (College of Agricultural Life Sciences, Cornell University, 2008).

9. Knighton, J., Pluer, E. M., Prestigiacomo, A. R., Effler, S. W. \& Walter, M. T. Topographic wetness guided dairy manure applications to reduce stream nutrient loads in Central New York, USA. J. Hydrol. Reg. 14, 67-82 (2017).

10. Sharara, M. et al. Spatially explicit methodology for coordinated manure management in shared watersheds. J. Environ. Manage. 192, 48-56 (2017).

11. Ribaudo, M. O., Gollehon, N. R. \& Agapoff, J. Land application of manure by animal feeding operations: Is more land needed?. J. Soil Water Conserv. 58(1), 30-38 (2003). 
12. Kassem, N., Sills, D., Posmanik, R., Blair, C. \& Tester, J. W. Combining anaerobic digestion and hydrothermal liquefaction in the conversion of dairy waste into energy: A techno economic model for New York state. Waste Manage. 103, 228-239 (2020).

13. Jahne, M. A., Rogers, S. W., Holsen, T. M., Grimberg, S. J. \& Ramler, I. P. Emission and dispersion of bioaerosols from dairy manure application sites: Human health risk assessment. Environ. Sci. Technol. 49(16), 9842-9849 (2015).

14. Ghafoori, E., Flynn, P. C. \& Feddes, J. J. Pipeline vs truck transport of beef cattle manure. Biomass Bioenergy 31(2-3), 168-175 (2007).

15. Font-Palma, C. Methods for the treatment of cattle manure-A review. C J. Carbon Res. 5(2), 27 (2018).

16. Zhang, R. H. \& Westerman, P. W. Solid-liquid separation of annual manure for odor control and nutrient management. Appl. Eng. Agric. 13, 385-393 (1997).

17. Møller, H. B., Lund, I. \& Sommer, S. G. Solid-liquid separation of livestock slurry: Efficiency and cost. Bioresour. Technol. 74, 223-229 (2000).

18. Burton, C. H. The potential contribution of separation technologies to the management of livestock manure. Livest. Sci. 112, 208-216 (2007).

19. Welsh, R., Grimberg, S., Gillespie, G. W. \& Swindal, M. Technoscience, anaerobic digester technology and the dairy industry: Factors influencing North Country New York dairy farmer views on alternative energy technology. Renew. Agric. Food Syst. 25(2), $170-180$ (2010).

20. Leytem, A. B., Dungan, R. S., Bjorneberg, D. L. \& Koehn, A. C. Emissions of ammonia, methane, carbon dioxide, and nitrous oxide from dairy cattle housing and manure management systems. J. Environ. Qual. 40(5), 1383-1394 (2011).

21. VanderZaag, A. et al. Potential methane emission reductions for two manure treatment technologies. Environ. Technol. 39(7), 1-22 (2017).

22. Lehmann, J. \& Joseph, S. Biochar for Environmental Management: Science, Technology and Implementation (Routledge, 2015).

23. Enders, A., Hanley, K., Whitman, T., Joseph, S. \& Lehmann, J. Characterization of biochars to evaluate recalcitrance and agronomic performance. Bioresour. Technol. 114, 644-653 (2012).

24. Ahmad, M. et al. Biochar as a sorbent for contaminant management in soil and water: A review. Chemosphere 99, 19-33 (2014).

25. Hollister, C. C., Bisogni, J. J. \& Lehmann, J. Ammonium, nitrate, and phosphate sorption to and solute leaching from biochars prepared from corn stover (Zea mays L.) and oak wood (Quercus spp.). J. Environ. Qual. 42(1), 137-144 (2013).

26. Mohan, D., Sarswat, A., Ok, Y. S. \& Pittman, C. U. Jr. Organic and inorganic contaminants removal from water with biochar, a renewable, low cost and sustainable adsorbent-A critical review. Bioresour. Technol. 160, 191-202 (2014).

27. Wang, B., Lehmann, J., Hanley, K., Hestrin, R. \& Enders, A. Ammonium retention by oxidized biochars produced at different pyrolysis temperatures and residence times. RSC Adv. 6(48), 41907-41913 (2016).

28. Shaaban, M. et al. A concise review of biochar application to agricultural soils to improve soil conditions and fight pollution. J. Environ. Manage. 228, 429-440 (2018).

29. Hestrin, R. et al. Fire-derived organic matter retains ammonia through covalent bond formation. Nat. Commun. 10, 664. https:// doi.org/10.1038/s41467-019-08401-z (2019).

30. Garner, W. The Disposal of Cattle Feedlot Wastes by Pyrolysis Vol. 1 (US Government Printing Office, 1973).

31. Schouten, S., van Groenigen, J. W., Oenema, O. \& Cayuela, M. L. Bioenergy from cattle manure? Implications of anaerobic digestion and subsequent pyrolysis for carbon and nitrogen dynamics in soil. Glob. Change Biol. Bioenerg. 4(6), 751-760 (2012).

32. Novak, J. M. \& Johnson, M. G. Elemental and spectroscopic characterization of low-temperature $\left(350^{\circ} \mathrm{C}\right)$ lignocellulosic-and manure-based designer biochars and their use as soil amendments. In Biochar from Biomass and Waste: Fundamentals and Applications (eds Ok, Y. S. et al.) 37-58 (Elsevier Inc, 2019).

33. Zhao, N., Lehmann, J. \& You, F. Poultry waste valorization via pyrolysis technologies: Economic and environmental life cycle optimization for sustainable bioenergy systems. ACS Sustain. Chem. Eng. 8(11), 4633-4646 (2020).

34. Struhs, E., Mirkouei, A., You, Y. \& Mohajeri, A. Techno-economic and environmental assessments for nutrient-rich biochar production from cattle manure: A case study in Idaho, USA. Appl. Energy 279, 115782 (2020).

35. Ro, K. S., Cantrell, K. B. \& Hunt, P. G. High-temperature pyrolysis of blended animal manures for producing renewable energy and value-added biochar. Ind. Eng. Chem. Res. 49(20), 10125-10131 (2010).

36. Cely, P., Gascó, G., Paz-Ferreiro, J. \& Méndez, A. Agronomic properties of biochars from different manure wastes. J. Anal. Appl. Pyrolysis 111, 173-182 (2015).

37. Wang, T., Arbestain, M. C., Hedley, M. \& Bishop, P. Chemical and bioassay characterisation of nitrogen availability in biochar produced from dairy manure and biosolids. Organ. Geochem. 51, 45-54 (2012).

38. Cantrell, K. B. et al. Impact of pyrolysis temperature and manure source on physicochemical characteristics of biochar. Bioresour. Technol. 107, 419-428 (2012).

39. Krounbi, L. et al. Biological and thermochemical conversion of human solid waste to soil amendments. Waste Manage. 89, 366-378 (2019).

40. Figueiredo, C. C. D. et al. Sewage sludge biochar increases nitrogen fertilizer recovery: Evidence from a $15 \mathrm{~N}$ tracer field study. Soil Use Manage. https://doi.org/10.1111/sum.12672 (2020).

41. Krounbi, L. et al. Sequential ammonia and carbon dioxide adsorption on pyrolyzed biomass to recover waste stream nutrients. ACS Sustain. Chem. Eng. 8(18), 7121-7131 (2020).

42. Fernandez-Lopez, M. et al. Life cycle assessment of swine and dairy manure: pyrolysis and combustion processes. Bioresour. Technol. 182, 184-192 (2015)

43. Taghizadeh-Toosi, A., Clough, T. J., Sherlock, R. R. \& Condron, L. M. Biochar adsorbed ammonia is bioavailable. Plant Soil 350 , 57-69 (2012).

44. Van Humbeck, J. F. et al. Ammonia capture in porous organic polymers densely functionalized with Brønsted acid groups. J. Am. Chem. Soc. 136, 2432-2440 (2014)

45. Shen, W. \& Fan, W. Nitrogen-containing porous carbons: Synthesis and application. J. Mat. Chem. A. 1(4), 999-1013 (2013).

46. Shafeeyan, M. S., Daud, W. M. A. W., Houshmand, A. \& Arami-Niya, A. Ammonia modification of activated carbon to enhance carbon dioxide adsorption: Effect of pre-oxidation. Appl. Surf. Sci. 257, 3936-3942 (2011).

47. Taghizadeh-Toosi, A., Clough, T. J., Sherlock, R. R. \& Condron, L. M. A wood based low-temperature biochar captures $\mathrm{NH}_{3}-\mathrm{N}$ generated from ruminant urine-N, retaining its bioavailability. Plant Soil 353, 73-84 (2012).

48. Environmental Protection Agency. A Guide to the Biosolids Risk Assessments for the EPA Part 503 Rule (US Environmental Protection Agency, Office of Wastewater Management, 1995).

49. Cárdenas-Aguiar, E., Gascó, G., Paz-Ferreiro, J. \& Méndez, A. Thermogravimetric analysis and carbon stability of chars produced from slow pyrolysis and hydrothermal carbonization of manure waste. J. Anal. Appl. Pyrolysis 140, 434-443 (2019).

50. Pettygrove, G. S. et al. Mineralization of nitrogen in dairy manure water. Western Nutr. Manag. Conf. 5, 34-41 (2003).

51. Grunert, O. et al. Growing media constituents determine the microbial nitrogen conversions in organic growing media for horticulture. Microb. Biotechnol. 9(3), 389-399 (2016).

52. Yeh, J. T., Resnik, K. P., Rygle, K. \& Pennline, H. W. Semi-batch absorption and regeneration studies for $\mathrm{CO}_{2}$ capture by aqueous ammonia. Fuel Process Technol. 86, 1533-1546 (2005).

53. Dutcher, B., Fan, M. \& Russell, A. G. Amine-based $\mathrm{CO}_{2}$ capture technology development from the beginning of 2013: A review. ACS Appl. Mat. Interfaces 7, 2137-2148 (2015). 
54. McLeod, A., Jefferson, B. \& McAdam, E. J. Biogas upgrading by chemical absorption using ammonia rich absorbents derived from wastewater. Water Res. 67, 175-186 (2014).

55. NY State Comptroller. A profile of agriculture in New York State. https:/www.osc.state.ny.us/files/reports/special-topics/pdf/agric ulture-report-2019.pdf (Office of Budget and Policy Analysis, Office of the New York State Comptroller, 2019).

56. Nennich, T. et al. Development of standard methods to estimate manure production and nutrient characteristics from dairy cattle. In IX 1-Animal, Agricultural and Food Processing Wastes (ed. Burns, R. T.) 263-268 (American Society of Agricultural and Biological Engineers, 2013). https://doi.org/10.13031/2013.15260.

57. Cornell University. Fertilizers for corn. https://fieldcrops.cals.cornell.edu/corn/fertilizers-corn/ (College of Agricultural and Life Sciences (CALS), 2021).

58. R Computing Team. $R$ Language Definition (R Foundation for Statistical Computing, 2000).

59. Wilkinson, L. ggplot2: Elegant graphics for data analysis by Wickham, H. Biometrics 67, 678-679 (2011)

60. Lenth, R. V. Least-squares means: The R package lsmeans. J. Stat. Softw. 69, 1-33 (2016).

61. Graves, S., Piepho, H. P., Selzer, L. \& Dorai-Raj, S. multcompView: Visualizations of paired comparisons. R package version 0.1-5; 46. https://CRAN.R-project.org/package=multcompView (2012).

\section{Acknowledgements}

This work was funded by the NSF IGERT Food Systems and Poverty Reduction (Grant 0903371), NSF-BREAD (Grant IOS-0965336, OPP51589), the Bradfield Research Award (Grant number 1258485), USDA Hatch (NYC125443, NYC-125314), and SARE (GNE17-154). Any opinions, findings and conclusions or recommendations expressed in this material are those of the authors and do not necessarily reflect the views of the donors.

\section{Author contributions}

L.K. and J.L. planned the experiments, L.K., A.E., M.B. and J.G. conducted the experiments. L.K. and J.L. wrote the first draft of the manuscript, all authors commented on and edited the manuscript.

\section{Competing interests}

LK, AE, and JL have no financial interests. JG is shareholder and MB is employee of bio365, a company that produces potting media. None of the investigated materials are currently sold as commercial products.

\section{Additional information}

Supplementary Information The online version contains supplementary material available at https://doi.org/ 10.1038/s41598-021-94337-8.

Correspondence and requests for materials should be addressed to J.L.

Reprints and permissions information is available at www.nature.com/reprints.

Publisher's note Springer Nature remains neutral with regard to jurisdictional claims in published maps and institutional affiliations.

(c) (i) Open Access This article is licensed under a Creative Commons Attribution 4.0 International License, which permits use, sharing, adaptation, distribution and reproduction in any medium or format, as long as you give appropriate credit to the original author(s) and the source, provide a link to the Creative Commons licence, and indicate if changes were made. The images or other third party material in this article are included in the article's Creative Commons licence, unless indicated otherwise in a credit line to the material. If material is not included in the article's Creative Commons licence and your intended use is not permitted by statutory regulation or exceeds the permitted use, you will need to obtain permission directly from the copyright holder. To view a copy of this licence, visit http://creativecommons.org/licenses/by/4.0/.

(C) The Author(s) 2021 